\title{
Electrostatic Mode Coupling of Beat-Excited Electron Plasma Waves
}

\author{
C. DARROW, W. B. MORI, T. KATSOULEAS, C. JOSHI, MEMBER, IEEE, D. UMSTADTER, \\ AND C. E. CLAYTON
}

\begin{abstract}
The process of beat excitation of electron plasma waves in a plasma containing a density ripple is studied theoretically, experimentally, and computationally. A simple theoretical model has been developed which, for modest experimental parameters, predicts a new beat wave saturation mechanism. This mechanism involves the excitation of a spectrum of secondary electrostatic modes which divert pump energy from the beat wave and can lead to saturation of the beat wave at an amplitude well below that expected for relativistic detuning. Experiments designed to study the coupled mode spectrum were performed. The measured properties of the electrostatic spectrum are in reasonable agreement with the theory. To bridge the gap between the idealized model and the experiment, computer simulations were performed for a variety of parameters. The results of the simulations are in excellent agreement with the theory at early times prior to the onset of purely kinetic effects. For later times the simulations exhibit qualitative behavior which is consistent with the experimental measurements. Under certain conditions the model predicts the thermal quenching of the mode coupling saturation mechanism. These predictions are also verified in the simulations.
\end{abstract}

\section{INTRODUCTION}

C MOLLECTIVE particle accelerator schemes rely on the large space-charge fields which arise when electrons are separated from ions to trap and accelerate charged particles to high energies [1], [2]. In the case of the plasma beat wave accelerator (PBWA) the electron plasma waves are created by the nonlinear force which arises when two collinear laser beams of slightly different frequencies beat in a plasma. This force causes longitudinal bunching of plasma electrons. The frequency and wavenumber of the bunching force equal the difference frequency and difference $k$ of the two lasers. If $\Delta \omega_{n l}, \Delta k_{n l}=\Delta \omega_{\text {lasers }}, \Delta k_{\text {lasers }}$ lies somewhere on the Bohm-Gross dispersion curve, plasma waves are resonantly driven by this force. The PBWA scheme exploits this resonance condition and the high fields attainable with present-day lasers to drive the

Manuscript received October 1, 1986. This work was supported by the U.S. Department of Energy under Contract DE-AT03-83ER40121, the National Science Foundation under Grant ECS 83-10972, the Lawrence Livermore National Laboratory University Research Program, and by the Los Alamos National Laboratory under University Grant 344-6905.

C. Darrow was with the Department of Physics, University of California, Los Angeles, Los Angeles, CA 90024. He is now with the Lawrence Livermore National Laboratory, Livermore, CA 94550.

W. B. Mori, T. Katsouleas, C. Joshi, and C. E. Clayton are with the Department of Electrical Engineering, University of California, Los Angeles, Los Angeles, CA 90024.

D. Umstadter is with the Department of Physics, University of California, Los Angeles, CA 90024.

IEEE Log Number 8613315. electron plasma waves to extremely large amplitudes for high-gradient particle acceleration. For efficient operation it is desirable to excite plasma wavefields which are as large as possible and which remain coherent over the entire acceleration length.

In this paper effects which make it difficult to meet these two conditions will be investigated. In particular, a mechanism which leads to saturation of the beat wave and disruption of its spatial coherence will be considered. This mechanism involves the scattering of the plasma beat wave by density fluctuations present in the plasma. Alternatively, this process can be thought of as mode coupling of the beat wave to larger $k$ electrostatic modes which, through Landau damping, can give rise to appreciable wave-plasma coupling. This mechanism is important to the PBWA because 1) the excitation of large- $k$ modes leads to a spatially incoherent accelerating field, and 2) the beat wave grows to a smaller amplitude because the driver energy is distributed among a large number of modes. Experimentally, the density fluctuations of interest are likely to be encountered when stimulated Brillouin scatter (SBS) driven ion waves are excited by either of the two incident laser beams. This work differs from previous work by other authors who have studied the evolution of resonantly excited plasma waves with the pump but without the ripple [3]-[6] and with the ripple but without the pump [7], [8].

In Section II we begin the study of this topic by reviewing the formulation of the beat wave problem. After reviewing the results of previous work that has treated beat wave excitation in uniform plasmas we present a model for beat excitation in a cold plasma containing a sinusoidal density ripple. We then go on to consider corrections for finite plasma temperature in preparation for interpretation of the experimental results. In Sections III and IV we present the results of our experimental studies of beat wave excitation and the coupled mode spectrum. In Section $\mathrm{V}$ we present the results of computer simulations which have been used to further corroborate the theoretical and experimental results.

\section{Theory of Beat Wave Excitation}

It is our purpose here to examine the plasma response to an excitation resulting from two beating laser beams. We began with the simple equation of motion for the elec- 
tron plasma wave electric field derived from the warm plasma field equations for a uniform plasma:

$$
\ddot{E}+\omega_{p}^{2}(x) E+c_{e}^{2} E^{\prime \prime}=\frac{1}{2} \alpha_{1} \alpha_{2} \omega_{p 0}^{2} \sin (\Delta k x-\Delta \omega t)
$$

where $\Delta \omega, \Delta k$ are the frequency and wavenumber differences of the two laser beams, $\alpha_{j}$ is the $j$ th laser beam field strength in $v_{\text {osc }} / c, c_{e}^{2}=3 T_{e} / m$, and $\omega_{p}$ is the background plasma frequency. Note that we have allowed for nonuniformities in the plasma density through $\omega_{p}(x)$. In treating plasmas containing density inhomogeneities we will only require that these inhomogeneities be such that an average density $n_{0}$ can be defined so that $\omega_{p}\left(n_{0}\right)=\omega_{p 0}$ $\cong \Delta \omega_{\text {lasers }}$.

Assuming a driver which "turns on" at $t=0$ and neglecting the thermal velocity $c_{e}$ for now, the solution to (1) can readily be shown to be

$$
\begin{aligned}
E(x, t)= & \frac{\omega_{p 0}^{2} F}{\omega_{p}^{2}-\omega_{p 0}^{2}}\left[\sin \left(k_{p} x-\omega_{p 0} t\right)\right. \\
& +\frac{\omega_{p 0}}{\omega_{p}} \sin \left(\omega_{p} t\right) \cos \left(k_{p} x\right) \\
& \left.-\cos \left(\omega_{p} t\right) \sin \left(k_{p} x\right)\right]
\end{aligned}
$$

where $F=\frac{1}{2} \quad \alpha_{1} \alpha_{2}$ parameterizes the beat wave driver strength and we now denote the driver $\omega$ and $k$ as $\Delta k=$ $k_{p}, \Delta \omega=\omega_{p 0}$.

\section{A. Uniform Plasma}

Exactly resonant beat wave excitation in the absence of damping leads to resonant growth of a plasma wave to an arbitrarily large amplitude. To study the behavior we therefore consider the solution of (2) for "near-resonant" excitation by writing $\omega_{p}=\omega_{p 0}+\delta \omega$ where $\delta \omega / \omega_{p 0}$ $\ll 1$. With this form for $\omega_{p}$, (2) can be written as

$$
\begin{aligned}
E(x, t)= & \frac{\omega_{p 0}^{2} F}{2 \omega_{p 0} \delta \omega}\left[\sin (\delta \omega t) \cos \left(k_{p} x-\omega_{p 0} t\right)\right. \\
& -\frac{\delta \omega}{\omega_{p}} \cos \left(k_{p} x\right)\left\{\cos (\delta \omega t) \sin \left(\omega_{p 0} t\right)\right. \\
& \left.\left.+\sin (\delta \omega t) \cos \left(\omega_{p 0} t\right)\right\}\right] .
\end{aligned}
$$

For $\delta \omega t \ll 1$ and $\delta \omega / \omega_{p 0} \ll 1$, sin $(\delta \omega t) \cong \delta \omega t$, cos $(\delta \omega t) \cong 1$, and $(3)$ simplifies to

$$
\begin{aligned}
E(x, t)= & \frac{\omega_{p 0} F}{2}\left[t \cos \left(k_{p} x\right) \cos \left(\omega_{p 0} t\right)\right. \\
& \left.-\frac{1}{\omega_{p 0}} \cos \left(k_{p} x\right) \sin \left(\omega_{p 0} t\right)\right] .
\end{aligned}
$$

For arbitrarily near-resonant excitation the beat wave field is seen to grow linearly in time (for $E(t=0)=0$ as the initial condition) with a growth rate $\gamma=$ $\alpha_{1} \alpha_{2} \omega_{p 0} / 4$. The growth continues to an arbitrarily large field amplitude until the assumption $\delta \omega t<1$ is violated. This behavior is modified when relativistic effects are included in the electron field equations. Rosenbluth and Liu [6] and others, by using a fully relativistic equation of motion for the electron fluid, have studied such effects. In the relativistic case the field grows linearly in time at early times with the same growth rate as given above. However, the field saturates abruptly upon reaching an amplitude $E=\left(16 \alpha_{1} \alpha_{2} / 3\right)^{1 / 3}$. Saturation occurs because of the relativistic electron mass increase incurred by the electrons as they attain relativistic quiver velocities in the plasma wavefield. This mass increase shifts the electron plasma frequency and detunes the electrons from the driver. This saturation limits the field amplitude of the beat wave and is therefore of importance in the PBWA scheme.

\section{B. Inhomogeneous Plasma: Density Ripple}

Equation (1) provides a means for the study of the evolution of the beat wave in inhomogeneous plasmas through $\omega_{p}(x)$. It has been shown that in homogeneous plasmas there exists a mechanism whereby the amplitude of the beat wave is limited [6]. We now consider the beat wave process in plasmas containing density inhomogeneities for the purpose of determining what other processes, if any, can limit the beat wave amplitude. We consider inhomogeneities or spatial fluctuations of the form

$$
n(x)=n_{0}\left(1+\epsilon \sin k_{i} x\right) \text {. }
$$

This form of an inhomogeneity is particularly interesting since 1) a sinusoidal ripple is easily excited under beat wave experimental conditions due to the cogeneration of low-frequency $\left(\omega_{a c} \ll \omega_{p}\right)$ SBS-driven ion acoustic waves, and 2) an arbitrary inhomogeneity can be decomposed into its spatial Fourier components. The understanding of how one such component scatters the beat wave gives insight into how the beat wave would be affected by an arbitrary inhomogeneity. In the development which follows we will be particularly interested in characterizing the growth and maximum amplitude of the beat wave.

Some insight into the problem can be gained prior to any further mathematical manipulations. We have already observed that the plasma responds to a driving force of frequency $\omega_{p 0}$ much as a simple harmonic oscillator of natural frequency $\omega_{p}$. The magnitude of the response depends on how well the frequencies of the oscillator and driver are matched. In a plasma containing a ripple $n=$ $n_{0}\left(1+\epsilon \sin k_{i} x\right)$, where $\omega_{p}\left(n_{0}\right)=\omega_{p 0}$, the response at the points along $x$ where $\sin \left(k_{i} x\right)=0$ will be large while the response at points where $\sin \left(k_{i} x\right)=1$ will be weaker. Other points in the profile will have some intermediate response. At some time $t$ the waveform $E(x)$ will have a variation in $x$ with one component of spatial periodicity 
$2 \pi / k_{i}$. Since $k_{\text {driver }}=\Delta k$ is small for waves with $v_{\phi} \cong$ $c$, the driver may be considered nearly uniform in space over many density ripple wavelengths, $\lambda_{i}=2 \pi / k_{i}$. Although the driver is nearly uniform in $x$, the resulting waveform develops spatial Fourier components with $k \cong$ $k_{i}$. Since the response is nonlinear in $\omega_{p}-\omega_{p 0}, E(x)$ does not simply follow $n(x)$ but instead has a complex $k$ spectrum whose components consist of the various spatial harmonics of $k_{i}$.

To rigorously treat the evolution of the beat wave excited in a plasma containing a density ripple, we return to the solution of (1) given by (2) and adopt the complex notation:

$$
E(x, t)=\frac{1}{2 i}[y(x, t)-\text { complex conjugate }]
$$

where

$$
\begin{aligned}
y(x, t)= & \frac{\omega_{p 0}^{2} F}{\left(\omega_{p}^{2}-\omega_{p 0}^{2}\right)}\left[\exp \left\{i\left(k_{p} x-\omega_{p 0} t\right)\right\}\right. \\
& -\exp \left\{i\left(k_{p} x-\omega_{p} t\right)\right\} \\
& -\frac{\omega_{p}-\omega_{p 0}}{2 \omega_{p}}\left[\exp \left\{i\left(k_{p} x+\omega_{p} t\right)\right\}\right. \\
& \left.\left.-\exp \left\{i\left(k_{p} x-\omega_{p} t\right)\right\}\right]\right]
\end{aligned}
$$

where as before $\omega_{p}=\omega_{p}(x), \omega_{p}\left(n_{0}\right)=\omega_{p 0}, F=\alpha_{1} \alpha_{2} / 2$, and $E=E_{1} / E_{\text {cold }}$. We consider small density ripples

$$
n(x)=n_{0}\left[1+\epsilon \sin k_{i} x\right], \quad \epsilon \ll 1
$$

so that

$$
\begin{aligned}
\omega_{p}(x)= & \omega_{p 0}\left[1+\epsilon \sin k_{i} x\right]^{1 / 2} \\
& \cong \omega_{p 0}\left[1+\frac{\epsilon}{2} \sin k_{i} x\right] .
\end{aligned}
$$

Substitution of this form of $\omega_{p}$ into (6) and rearranging terms yields

$$
\begin{aligned}
y= & \frac{\omega_{p 0}^{2} F}{\left(\omega_{p}^{2}-\omega_{p 0}^{2}\right)} \exp \left\{i\left(k_{p} x-\omega_{p 0} t\right)\right\} \\
& \times\left[1-\exp \left\{-i \frac{\epsilon \omega_{p 0} t}{2} \sin k_{i} x\right\}+\frac{\epsilon}{4} \sin k_{i} x\right. \\
& \times\left[\exp \left\{-i \frac{\epsilon \omega_{p 0} t}{2} \sin k_{i} x\right\}\right. \\
& \left.\left.-\exp \left\{i \omega_{p 0} t\left(2+\frac{\epsilon}{2} \sin k_{i} x\right)\right\}\right]\right] .
\end{aligned}
$$

Writing $-i\left(\epsilon \omega_{p 0} t / 2\right) \sin k_{i} x=i\left(\epsilon \omega_{p 0} t / 2\right) \sin \left(-k_{i} x\right)$ and applying the Bessel identity

$$
\exp \{i x \sin \theta\}=\sum_{n=-\infty}^{+\infty} J_{n}(x) \exp \{\operatorname{in} \theta\}
$$

(9) becomes

$$
\begin{aligned}
y= & \frac{\omega_{p 0}^{2} F}{\left(\omega_{p}^{2}-\omega_{p 0}^{2}\right)}\left[e^{i\left(k_{p} x-\omega_{p 0} t\right)}-\sum_{n=-\infty}^{+\infty} J_{n}\left(\frac{\epsilon \omega_{p 0} t}{2}\right)\right. \\
& \cdot \exp \left\{i\left[\left(k_{p}-n k_{i}\right) x-\omega_{p 0} t\right]\right\} \\
& +\frac{\epsilon}{4} \sum_{n=-\infty}^{+\infty} J_{n}\left(\frac{\epsilon \omega_{p 0} t}{2}\right) \sin k_{i} x \\
& \cdot\left[\exp \left\{i\left[\left(k_{p}-n k_{i}\right) x-\omega_{p 0} t\right]\right\}\right. \\
& \left.\left.-\exp \left\{i\left[\left(k_{p}+n k_{i}\right) x+\omega_{p 0} t\right]\right\}\right]\right] .
\end{aligned}
$$

From the definition of $E$ in (5), it follows that the electric field can be written as

$$
\begin{aligned}
E(x, t)= & \frac{\omega_{p 0}^{2} F}{\left(\omega_{p}^{2}-\omega_{p 0}^{2}\right)}\left[\sin \Psi_{0}-\sum_{n=-\infty}^{+\infty} J_{n}\left(\frac{\epsilon \omega_{p 0} t}{2}\right)\right. \\
& \cdot \sin \left(\Psi_{0}-n k_{i} x\right)+\frac{\epsilon}{4} \sum_{n=-\infty}^{+\infty} J_{n}\left(\frac{\epsilon \omega_{p 0} t}{2}\right) \\
& \cdot \sin k_{i} x\left[\sin \left(\Psi_{0}-n k_{i} x\right)-\sin \left(\bar{\Psi}_{0}\right.\right. \\
& \left.\left.\left.+n k_{i} x\right)\right]\right]
\end{aligned}
$$

where $\Psi_{0}=k_{p} x-\omega_{p 0} t$ and $\bar{\Psi}_{0}=k_{p} x+\omega_{p 0} t$. Noting that $\omega_{p}^{2}-\omega_{p 0}^{2}=\epsilon \omega_{p 0}^{2} \sin k_{i} x$, the electric field $E$ simplifies to

$$
\begin{aligned}
E(x, t)= & \frac{F}{\epsilon}\left[\frac{\sin \Psi_{0}}{\sin k_{i} x}-\sum_{n=-\infty}^{+\infty} J_{n}\left(\frac{\epsilon \omega_{p 0} t}{2}\right) \frac{\sin \left(\Psi_{0}-n k_{i} x\right)}{\sin k_{i} x}\right. \\
& +\frac{\epsilon}{4} \sum_{n=-\infty}^{+\infty} J_{n}\left(\frac{\epsilon \omega_{p 0} t}{2}\right)\left[\sin \left(\Psi_{0}-n k_{i} x\right)\right. \\
& \left.-\sin \left(\bar{\Psi}_{0}+n k_{i} x\right)\right] .
\end{aligned}
$$

Equation (10) shows the complex electrostatic $\omega-k$ spectrum which arises when plasma waves are "resonantly" excited in a rippled density plasma. For the purpose of comparison with experimental results it is of interest to cast (10) into the form

$$
E(x, t)=\sum_{m} a_{m}(t) \exp \left(i \Psi_{m}\right)
$$

where

$$
\Psi_{m}=i\left[\left(k_{p}+m k_{i}\right) x-\omega_{p 0} t\right] .
$$

In (3) the coefficients $a_{j}$ describe the slow-time behavior of the various spatial "harmonics" $\Psi_{m}\left(k_{p}+m k_{i} \cong m k_{i}\right.$ since $k_{p}<<k_{i}$ ) of the plasma wave electric field.

To explicitly project out the various $\Psi_{m}$ components, we expand $1 / \sin k_{i} x$ in (10) in a Fourier series:

$$
\frac{1}{\sin k_{i} x}=\sum_{m=0}^{\infty} 2 \sin \left\{(2 m+1) k_{i} x\right\} .
$$

Applying a trigonometric identity and rearranging terms yields 


$$
\begin{aligned}
E(x, t)= & \frac{F}{\epsilon} \sum_{m=0}^{\infty}\left[\cos \left\{\Psi_{0}-(2 m+1) k_{i} x\right\}\right. \\
& \left.-\cos \left\{\Psi_{0}+(2 m+1) k_{i} x\right\}\right] \\
& -\frac{F}{\epsilon} \sum_{m=0}^{\infty} \sum_{n=-\infty}^{+\infty} J_{n}\left(\frac{\epsilon \omega_{p 0} t}{2}\right) \\
& \cdot\left[\cos \left\{\Psi_{0}-\left[n+(2 m+1) k_{i} x\right]\right\}\right. \\
& \left.-\cos \left\{\Psi_{0}-\left[n-(2 m+1) k_{i} x\right]\right\}\right] \\
& +\frac{F}{4} \sum_{n=-\infty}^{+\infty} J_{n}\left(\frac{\epsilon \omega_{p 0} t}{2}\right)\left[\cos \left\{\Psi_{0}-n k_{i} x\right\}\right. \\
& \left.-\cos \left\{\bar{\Psi}_{0}+n k_{i} x\right\}\right] .
\end{aligned}
$$

The $a_{j}(t)$ coefficients are obtained by combining those terms in the sums of (13) for which $n \pm(2 m+1)=j$ and $(2 m+1)=j$ :

\section{$\Psi_{0}$ (beat wave) component:}

First term: No contribution.

Second term: Consider $m=0,1$ and $n= \pm 1, \pm 2$, and \pm 3 :

$$
\begin{array}{ll}
m=0 & n=+1:\left[\cos \left(\Psi_{0}-2 k_{i} x\right)-\cos \left(\Psi_{0}\right)\right] J_{+1} \\
m=0 & n=-1:\left[\cos \left(\Psi_{0}\right)-\cos \left(\Psi_{0}+2 k_{i} x\right)\right] J_{-1} \\
m=1 & n=+3:\left[\cos \left(\Psi_{0}-6 k_{i} x\right)-\cos \left(\Psi_{0}\right)\right] J_{+3} \\
m=1 & n=-3:\left[\cos \left(\Psi_{0}\right)-\cos \left(\Psi_{0}+6 k_{i} x\right)\right] J_{-3} .
\end{array}
$$

Third term: Standing wave terms.

Combining the $\Psi_{0}$ terms one finds

$$
E\left(\Psi_{0}\right)=\frac{2 F}{\epsilon} \sum_{n=1 \text { (odd) }}^{\infty} J_{n}\left(\frac{\epsilon \omega_{p 0} t}{2}\right) .
$$

Similarly for the $\Psi_{+1}$ component:

$\Psi_{+1}$ ( first coupled mode) component:

First term: $m=0:-\cos \left(\Psi_{1}\right)$.

Second term: Consider $m=0,1,2,3$ and $n= \pm 2$, $\pm 4, \pm 6, \pm 8$ :

$$
m=0 \quad n=-2: J_{-2} \cos \left(\Psi_{1}\right) \quad n=0:-J_{0} \cos \left(\Psi_{1}\right)
$$$$
m=1 \quad n=-4: J_{-4} \cos \left(\Psi_{1}\right) \quad n=2:-J_{2} \cos \left(\Psi_{1}\right)
$$$$
m=2 \quad n=-6: J_{-6} \cos \left(\Psi_{1}\right) \quad n=4:-J_{4} \cos \left(\Psi_{1}\right)
$$$$
m=3 \quad n=-8: J_{-8} \cos \left(\Psi_{1}\right) \quad n=8:-J_{6} \cos \left(\Psi_{1}\right) \text {. }
$$

Since $J_{2 n}=J_{-2 n}$ one finds

$$
E\left(\Psi_{1}\right)=\frac{F}{\epsilon}\left[J_{0}\left(\frac{\epsilon \omega_{p 0} t}{2}\right)-1\right] \cos \left(\Psi_{1}\right) .
$$

In the same way, the temporal coefficients of the higher $k$ components follow. In the notation of (3) we can summarize the results for $\Psi_{0}, \Psi_{1}, \Psi_{2}$, and $\Psi_{3}$ :

$$
\begin{aligned}
\Psi_{0}: a_{0} & =\frac{2 F}{\epsilon} \sum_{n=1(\text { odd })}^{\infty} J_{n}\left(\frac{\epsilon \omega_{p 0} t}{2}\right) \\
& =\frac{2 F}{\epsilon} \int_{0}^{t} J_{0}\left(\frac{\epsilon \omega_{p 0} t^{\prime}}{2}\right) d\left(\frac{\epsilon \omega_{p 0} t^{\prime}}{2}\right)
\end{aligned}
$$

(a)
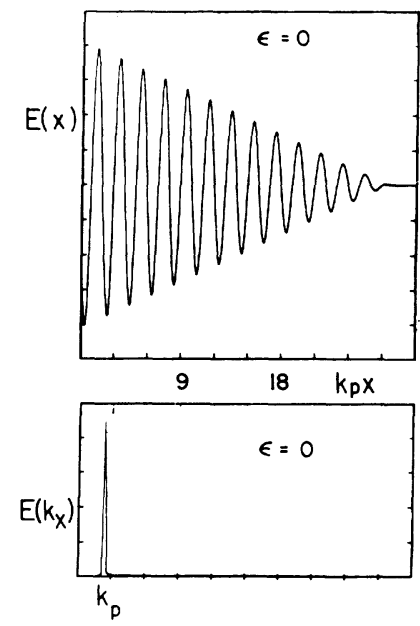

(b)
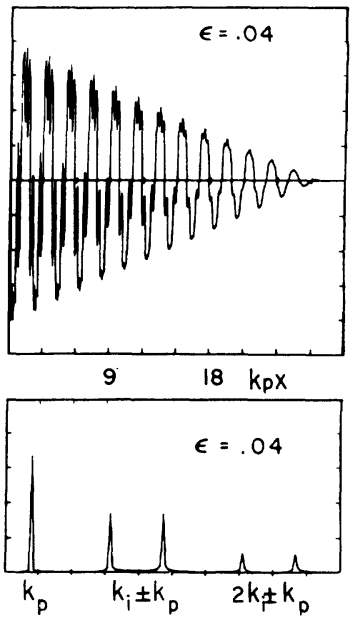

Fig. 1. Spatial evolution of the electric field with $(\epsilon=0.04)$ and without $(\epsilon=0)$ the ripple from the numerical solution of (1) with $\alpha_{1} \alpha_{2}=3 \times$ $10^{-4}, c_{e}=0$, and $k_{i} / k_{p}=5$. The spectra are taken at the left-hand side of the box. (a) No ripple: $\epsilon=0$. (b) With a ripple: $\epsilon=0.04$.

$$
\begin{aligned}
\Psi_{ \pm 1}: a_{ \pm 1}= & \mp \frac{F}{\epsilon}\left[1-J_{0}\left(\frac{\epsilon \omega_{p 0} t}{2}\right)\right] \\
\Psi_{ \pm 2}: a_{ \pm 2}= & \mp \frac{2 F}{\epsilon}\left[J_{3}\left(\frac{\epsilon \omega_{p 0} t}{2}\right)+J_{5}\left(\frac{\epsilon \omega_{p 0} t}{2}\right)\right. \\
& \left.+J_{7}\left(\frac{\epsilon \omega_{p 0} t}{2}\right)+\cdots\right] \\
\Psi_{ \pm 3}: a_{ \pm 3}= & \mp \frac{F}{\epsilon}\left[1-J_{0}\left(\frac{\epsilon \omega_{p 0} t}{2}\right)-2 J_{2}\left(\frac{\epsilon \omega_{p 0} t}{2}\right)\right] .
\end{aligned}
$$

The results presented in (16a)-(16d) can be verified by numerically calculating the exact solution of (1) given by (2) and then examining its Fourier $k$ spectrum.

In Fig. 1 we have plotted the numerical solution of (1) with $(\epsilon=0.04)$ and without $(\epsilon=0)$ the density ripple. The beat wave driver is incident from the left and has just reached the right-hand boundary. For the homogeneous plasma case $(\epsilon=0)$ a coherent beat wave is seen to grow secularly in time (note the linear ramping in $x$ since the back edge of the pulse has had longer time to grow) and the Fourier spectrum shows a single mode at $k=k_{p}$ as expected. In the rippled plasma case $(\epsilon=0.04)$ higher $k$ components are also excited and, although the peak amplitude of the waveform still exhibits secular growth with the same growth rate, the relative size of the beat wave component $\left(\Psi_{0}\right)$ is reduced.

In Fig. 2 we have plotted the temporal evolution of the first three $k$ components $\left(a_{0}, a_{ \pm 1}\right.$, and $\left.a_{ \pm 2}\right)$ of the exact solution, (2). For the early times shown, the exact solutions of Fig. 2 match the small $\epsilon \omega_{p 0} t / 2$ expressions ((16a)-(16c)) nearly exactly.

Referring to Fig. 2 it is seen that the two-frequency pump excites the beat wave ( $\left.\Psi_{0}\right)$ component first. However, the beat wave rapidly saturates as wave energy is coupled out of the beat wave and into the first coupled 
(a)

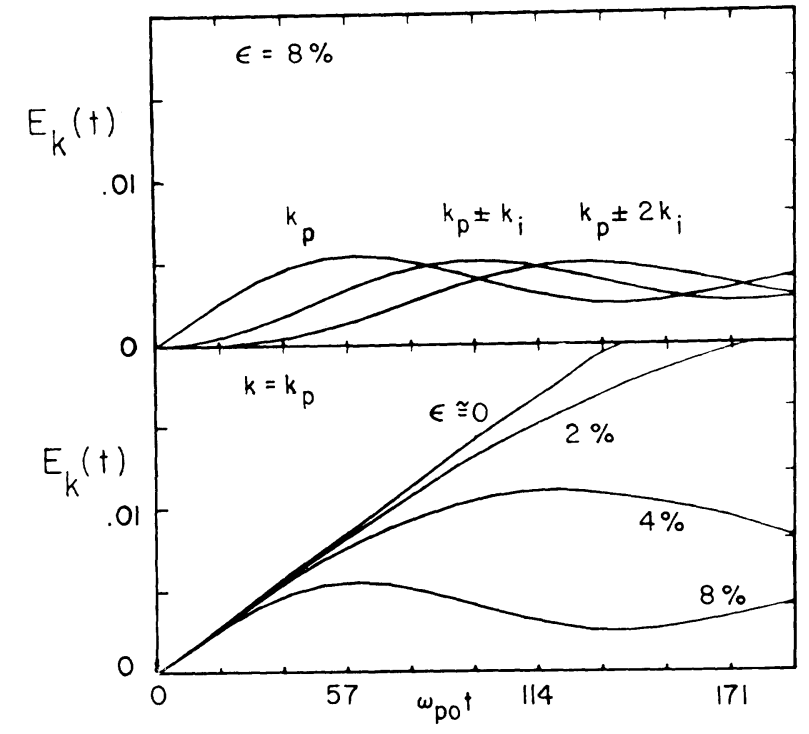

Fig. 2. Temporal evolution of the beat wave and coupled modes with $\alpha_{1} \alpha_{2}$ $=3 \times 10^{-4}$. (a) Evolution of beat wave and first two coupled modes ( $\Psi_{m}, m=0, \pm 1, \pm 2$ ) for $\epsilon=0.08$. (b) Temporal evolution of beat wave for several ripple amplitudes $\epsilon$.

mode $\left(\Psi_{ \pm 1}\right)$. The first coupled mode begins to grow but then saturates as the second coupled mode is excited, and so on. For the above cold-plasma treatment the coupling proceeds indefinitely to higher and higher $k$ modes.

One should note the limitations of the above formalism due to two approximations which were made in its development. First we neglected higher-than-first-order terms when we expanded $\omega_{p}(x)$ in (8). While it is reasonable to assume $\epsilon<<1$, it is still possible that a significant contribution to the exponential phase can be made in (9) if $\omega_{p 0} t \epsilon^{2} / 8 \cong \pi / 2$. Thus the validity of our results is limited to $\omega_{p 0} t<2.5 / \epsilon^{2}$ or, in the worst case in our work, $\omega_{p 0} t \leq 170$. Secondly, we neglected higher order terms in the plasma wave amplitude compared to the first-order ripple correction. That is, we were implicitly assuming terms of order $E^{2}$ were much smaller than terms of order $\epsilon\left(E^{2}<\epsilon \epsilon\right)$. This assumption is justified for the parameters of interest in this work. Furthermore, the validity of neglecting nonlinear plasma wave terms was verified by the numerical solution of the exact Lagrangian equations for the driven cold fluid oscillations:

$$
\begin{gathered}
x=x_{0}+\xi\left(x_{0}, t\right) \\
{\left[\frac{\partial^{2}}{\partial t^{2}}+\omega_{p 0}^{2}\left(1+\epsilon \sin k_{i}\left(x_{0}+\xi\right)\right)\right] k_{p} \xi} \\
=F \sin \left(k_{i}\left(x_{0}+\xi\right)-\omega_{p 0} t\right) \\
E(x, t)=k_{p} \int_{0}^{\xi}\left(1+\epsilon \sin k_{i}\left(x_{0}+\xi^{\prime}\right)\right) d \xi^{\prime}
\end{gathered}
$$

where $F=\frac{1}{2} \alpha_{1} \alpha_{2}$ as before and $\xi$ is the displacement of a Lagrangian fluid element from its unperturbed position $x_{0}$. The second two equations are, respectively, the equation of motion and Poisson's equation.

\section{Beat Wave Saturation by Mode Coupling}

One of the most significant results of this analysis which is of particular interest to the PBWA is the rapid saturation of the beat wave field amplitude as exhibited in (16a) and in Fig. 2. This saturation can limit the effectiveness of the beat wave acceleration mechanism. Recall (16a):

$$
a_{0}=\frac{2 F}{\epsilon} \sum_{n=1 \text { (odd) }}^{\infty} J_{n}\left(\frac{\epsilon \omega_{p 0} t}{2}\right)=\frac{2 F}{\epsilon} S
$$

where

$$
\begin{aligned}
& S=\sum_{n=1(\text { odd })}^{\infty} J_{n}(x) \\
& x=\frac{\epsilon \omega_{p 0} t}{2} .
\end{aligned}
$$

The beat wave saturates when $S$ reaches its maximum:

$$
\begin{aligned}
\left.\frac{\partial S}{\partial x}\right|_{x_{0}} & =0=\sum_{n=1(\text { odd })}^{\infty} J_{n}^{\prime}\left(x_{0}\right)=\frac{1}{2} \sum_{n=1(\text { odd })}^{\infty}\left(J_{n-1}-J_{n+1}\right) \\
& =\frac{1}{2}\left[\left(J_{0}+J_{2}+\cdots\right)-\left(J_{2}+J_{4}+\cdots\right)\right] \\
& =\frac{1}{2} J_{0}\left(x_{0}\right) .
\end{aligned}
$$

Thus $S$ has its extremum at $x_{0}$ where $x_{0}=2.3937 \cdots$ is the first zero of $J_{0}$. Using the identity

$$
\sum_{n=1 \text { (odd) }}^{\infty} J_{n}\left(x_{0}\right)=\sum_{n=0}^{\infty} J_{2 n+1}\left(x_{0}\right)=\frac{1}{2} \int_{0}^{x_{0}} J_{0}(x) d x
$$

and performing the integration numerically yields $S_{\max }=$ 0.735 and

$$
E\left(\Psi_{0}, \text { saturation }\right)=0.735 \frac{2 F}{\epsilon}
$$

with $\tau_{\text {sat }}\left(\Psi_{0}\right) \cong 4.8\left(\epsilon \omega_{p}\right)^{-1}$. Similarly, it is found that

$$
E\left(\Psi_{ \pm 1}, \text { saturation }\right)=0.701 \frac{2 F}{\epsilon}
$$

with $\tau_{\text {sat }}\left(\Psi_{ \pm 1}\right) \cong 7.7\left(\epsilon \omega_{\rho}\right)^{-1}$.

Before comparing the characteristics of beat wave saturation for mode coupling and relativistic detuning, we consider the effects of finite electron temperature on the mode coupling beat wave saturation mechanism.

\section{Thermal Effects}

Mode coupling brings about a saturation of the beat wave because energy which is pumped into the primary (beat wave) mode couples rapidly to an infinite number of higher $k$ modes. When the rate at which energy is coupled to these secondary modes equals the rate at which driver energy is supplied to the primary mode, the primary mode saturates; i.e., the beat wave saturates.

The introduction of a finite temperature alters the previous estimates of the mode coupling saturation times and amplitudes. Because the frequency difference between the 
primary mode and the $m$ th coupled mode increases with $m$ (due to the finite Bohm-Gross frequency shift) only a finite number of secondary modes are excited to appreciable amplitudes since the maximum frequency difference allowed is equal to the variation of $\omega_{p}(x)$ within the density ripple. The larger the Bohm-Gross shift or the smaller the ripple depth $\epsilon$, the smaller the number of eligible secondary modes [7], [8].

The effect of finite temperature on the saturation is due to the finite number of coupled modes and the coupling efficiency for the off-resonance modes. Since primary mode energy has fewer avenues for depletion, the primary (beat wave) grows to a larger amplitude before saturating. We can also expect a longer saturation time.

To take thermal effects into consideration analytically we recall that the cold-plasma solution can be written as

$$
E(x, t)=\sum_{m} a_{m}(t) \exp \left\{i\left(k_{p}-m k_{i}\right) x-\omega_{p 0} t\right\} .
$$

In the case of a warm plasma we have argued that only a finite number of secondary modes are eligible for coupling. We therefore approximate $E(x, t)$ by truncating the summation in (18). For simplicity we allow only the first coupled modes $(n= \pm 1)$ :

$$
E_{\mathrm{warm}}=a_{0} e^{i \Psi_{0}}+a_{+1} e^{i \Psi+1}+a_{-1} e^{i \Psi-1}+\text { c.c. }
$$

where again, $\Psi_{0}=k_{p} x-\omega_{p 0} t$ and $\Psi_{ \pm 1}=\left(k_{p} \pm k_{i}\right) x-$ $\omega_{p 0} t$. Upon substitution of this form of a solution into the wave equation for $E(x, t)$ (see (1))

$$
\begin{gathered}
\ddot{E}+\omega_{p 0}^{2}\left(1+\epsilon \sin k_{i} x\right) E+c_{e}^{2} E^{\prime \prime} \\
=F \omega_{p 0}^{2} \sin \left(k_{p} x-\omega_{p 0} t\right)
\end{gathered}
$$

we obtain three equations for the three $a$ coefficients above:

$$
\begin{array}{r}
\dot{a}_{0}+\frac{\epsilon}{4}\left(a_{-1}-a_{+1}\right)-\frac{\left(c_{e} k_{p} / \omega_{p}\right)^{2}}{2 i} a_{0}-\frac{F}{4}=0 \\
\dot{a}_{+1}-\frac{1}{2 i}\left[c_{e}\left(k_{p}+k_{i}\right) / \omega_{p}\right]^{2} a_{+1}+\frac{\epsilon}{4} a_{0}=0
\end{array}
$$

and

$$
\dot{a}_{-1}-\frac{1}{2 i}\left[c_{e}\left(k_{p}-k_{i}\right) / \omega_{p}\right]^{2} a_{-1}-\frac{\epsilon}{4} a_{0}=0 .
$$

Equations (19a)-(19c) govern the slow-time behavior of the beat wave and first two coupled modes in a warm plasma. To determine the temporal evolution of the beat wave and coupled modes in a finite-temperature plasma containing a density ripple we proceed to decouple (19a)(19c) and solve for $a_{0}$ and $a_{ \pm 1}$. Differentiating (19a) with respect to time and substituting (19b) and (19c) we obtain an equation for $a_{0}$ :

$$
\begin{aligned}
\ddot{a}_{0}- & \dot{a}_{0}\left(k_{i} / 2 i\right)^{2}+a_{0} \frac{\epsilon^{2}}{8}\left[1-2\left(k_{i} / \epsilon\right)^{2}\left(k_{p} / k_{i}\right)^{2}\right] \\
+ & \frac{F k_{i}^{2}}{8}=\frac{F}{4} \delta(t)
\end{aligned}
$$

where we have used the normalization $\dot{a}_{0}=\partial a / \partial\left(\omega_{p 0} t\right)$ and the $k$ 's appearing in (20) have been normalized to $\omega_{p 0} / c_{e}$ (i.e., $k_{p}$ is really $k_{p} c_{e} / \omega_{p 0}$ ). Similarly, one finds for the coupled modes

$$
\dot{a}_{ \pm 1}-\frac{1}{2 i}\left(k_{p} \pm k_{i}\right)^{2} a_{ \pm 1}=\mp \frac{\epsilon}{4} a_{0} .
$$

The $\delta$-function driver arises when one differentiates the driver force $F$ which is represented by a step function turning on at $t=0$. The effect of this $\delta$-function driver on $a_{0}$ is that $a_{0}$ receives an impulsive "kick" at $t=0$ such that $\dot{a}_{0}(0)=F / 4$ while maintaining $a_{0}(0)=0$. Defining $a=\left(k_{i} / 2\right)^{2}$ and keeping only the lowest order terms in $k_{p} / k_{i}$, (20a) and (20b) become

$$
\begin{aligned}
\ddot{a}_{0}+2 i a \dot{a}_{0}+\frac{\epsilon^{2}}{8} a_{0} & =\frac{F}{4} \delta(t)-\frac{F a}{2 i} \\
\dot{a}_{ \pm 1}-\frac{1}{2 i} k_{i}^{2} a_{ \pm 1} & =\mp \frac{\epsilon}{4} a_{0} .
\end{aligned}
$$

Noting that the particular solution of (21a) differs from the homogeneous solution only by the constant $F a / 2 i$ (time-independent driver for arbitrary times), one can first solve for $a_{0}$ subject to the initial conditions above and then substitute $a_{0}$ into (21b) and obtain $a_{ \pm 1}$. One finds

$$
\begin{aligned}
a_{0}= & \frac{4 i F a}{\epsilon^{2}}\left[1-\left[\frac{c+1}{2 c}+\frac{\epsilon^{2}}{32 a^{2} c}\right] e^{-i a(1-c) t}\right. \\
& \left.-\left[\frac{c-1}{2 c}-\frac{\epsilon^{2}}{32 a^{2} c}\right] e^{-i a(1+c) t}\right] \\
a_{ \pm 1}= & \frac{\mp F}{2 \epsilon}\left[1-\left[\frac{c+1}{2 c}\right] e^{-i a(1-c) t}\right. \\
& \left.-\left[\frac{c-1}{2 c}\right] e^{-i a(1+c) t}\right]
\end{aligned}
$$

where $c=\left(1+2 \epsilon^{2} / k_{i}^{2}\right)^{1 / 2}=\left(1+\epsilon^{2} / 8 a^{2}\right)^{1 / 2}$ and, as before, $a=\left(k_{i} / 2\right)^{2}$. Recalling that the beat wave and first coupled mode components are given by

$$
\begin{aligned}
& E_{b w}=a_{0} e^{i \Psi_{0}}+a_{0}^{*} e^{-i \Psi_{0}} \\
& E_{ \pm 1}=a_{ \pm 1} e^{i \Psi_{ \pm 1}}+a_{ \pm 1}^{*} e^{-i \Psi_{ \pm 1}}
\end{aligned}
$$

one can write

$$
\begin{aligned}
E_{0}= & \frac{-2 F p}{\epsilon}\left[\sin \Psi_{0}-C_{1} \sin \left(\Psi_{0}-B_{1} t\right)\right. \\
& \left.-C_{2} \sin \left(\Psi_{0}-B_{2} t\right)\right] \\
E_{ \pm 1}= & \frac{\mp F}{\epsilon}\left[\cos \Psi_{ \pm 1}-A_{1} \cos \left(\Psi_{ \pm 1}-B_{1} t\right)\right. \\
& \left.-A_{2} \cos \left(\Psi_{ \pm 1}-B_{2} t\right)\right]
\end{aligned}
$$

where

$$
A_{1}=\frac{c+1}{2 c}, \quad A_{2}=\frac{c-1}{2 c}
$$




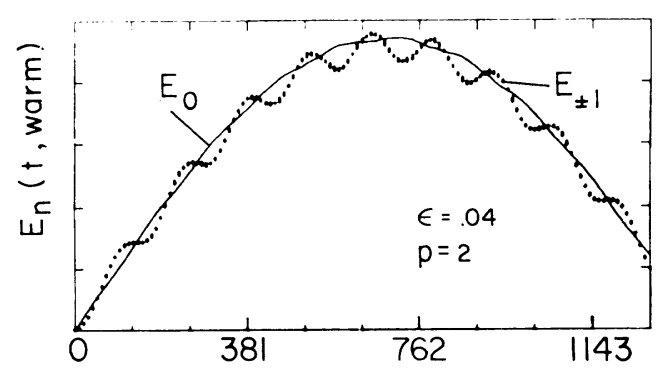

Fig. 3. Temporal evolution of the beat wave and first coupled mode from the warm-plasma results of (23) and (24) for $\alpha_{1} \alpha_{2}=3 \times 10^{-4}, \epsilon=$ 0.04 , and $p=2$. The $\Psi_{0}$ component is plotted on a different scale and at saturation reaches roughly twice the amplitude of the first coupled mode for these parameters.

$$
\begin{array}{ll}
B_{1}=\frac{\epsilon p}{4}(1-c), & B_{2}=\frac{\epsilon p}{4}(1+c) \\
C_{1}=A_{1}+\frac{1}{2 c p^{2}}, & C_{2}=A_{2}-\frac{1}{2 c p^{2}}
\end{array}
$$

and $p$ is a thermal parameter defined by $p=$ $3\left(k_{i} c_{e} / \omega_{p}\right)^{2} / \epsilon=3\left(k_{i} \lambda_{d}\right)^{2} / \epsilon$. Equations (23a) and (23b) describe the temporal evolution of the beat wave and first coupled modes in a finite temperature plasma. These results are presented in Fig. 3 where we have shown the temporal evolution of the beat wave and first coupled modes for one value of the thermal parameter $p$. In the cold plasma limit of the warm plasma model the beat wave and first coupled modes are seen to oscillate in time as energy is coupled back and forth between the three plasma modes ( $\Psi_{0}$ and $\left.\Psi_{ \pm 1}\right)$ and the pump. As expected, beat wave saturation is observed to occur. For warm plasmas $(p>0)$ the beat wave saturates at higher amplitudes and at later times due to weaker coupling induced by the increase with temperature of the Bohm-Gross frequency mismatch between the fixed frequency driver at $\omega_{p 0}$ and the coupled modes. The coupled modes are observed to attain lower amplitudes for the same reason.

As in the case of cold plasmas energy supplied by the pump is distributed among the beat wave and coupled modes $(n= \pm 1)$. This diversion of pump energy from the beat wave again leads to beat wave saturation. From (23a) and (23b) the warm plasma beat wave saturation amplitude and saturation time are found to be

$$
\begin{aligned}
E_{b w}(\text { sat }) & =\frac{2 F}{\epsilon} \times f(p), \\
f(p) & =\left[p+\left(1+p^{2}\right)\left(2+p^{2}\right)^{-1 / 2}\right]
\end{aligned}
$$

$\omega_{p 0} t_{\text {sat }}$ (beat wave, warm $) \cong 12 p / \epsilon, \quad$ for $p \geq 0.05$

$$
\begin{aligned}
E_{ \pm 1}(\text { sat }) & =\frac{2 F}{\epsilon} \\
\omega_{p 0} t_{\text {sat }}\left(\Psi_{ \pm 1}, \text { warm }\right) & \cong 12 p / \epsilon .
\end{aligned}
$$

One can see that the beat wave saturation amplitude is enhanced by the factor $f(p) \geq 2$ over that expected in cold plasma (17a).
A reasonable question at this point is, to what extent the saturation amplitude estimate provided by (25) is valid since the assumption of coupling only to the $m= \pm 1$ modes seems somewhat arbitrary. Clearly, in an experiment one would expect the parameters to be such that an exact treatment would lie somewhere between the two extremes (cold and warm) considered here. The warm plasma result of (25) can be checked in the limit of $p$ approaching zero (cold plasma). It is easily shown that

$$
\lim _{p \rightarrow 0} E_{b w}(\text { warm, sat })=0.707 \frac{2 F}{\epsilon} \text { with } \tau_{\text {sat }}=\left(\epsilon \omega_{p 0}\right)^{-1}
$$

in excellent agreement with the cold-plasma estimate of (17a). This remarkable agreement is due to the fact that, at the time of saturation of the beat wave, the higher order coupled modes ( $m>1$ ) have had insufficient time to grow to an appreciable amplitude and therefore contribute little to the total electric field as is evident in Fig. 2. This result justifies the truncation used in obtaining (26), since $p=$ 0 provides a worst case check of this approximation.

\section{E. Relative Importance of Saturation by Mode Coupling}

The relative importance of mode coupling as a beat wave saturation mechanism can be assessed by comparing the beat wave amplitude at saturation for the mode coupling and relativistic detuning cases. Recalling the beat wave saturation amplitudes for these two cases [5]:

$$
\begin{aligned}
\text { Relativistic Detuning: } E_{b w}(\text { sat }) & =\left[\frac{16}{3} \alpha_{1} \alpha_{2}\right]^{1 / 3} \\
\text { Mode Coupling: } E_{b w}(\text { sat }) & =\frac{\alpha_{1} \alpha_{2}}{\epsilon} f(p)
\end{aligned}
$$

it can be seen that saturation by mode coupling dominates over that due to relativistic detuning whenever

$$
\alpha_{1} \alpha_{2}<[1.6 \epsilon / f(p)]^{3 / 2}
$$

For example, in the case of a modest ripple size of $\epsilon \cong 4$ percent and a moderately warm plasma of $p \cong 2$, it is seen that saturation by mode coupling dominates unless $\alpha$ $>0.04$ (assuming equal laser line intensities, $\alpha_{1}=\alpha_{2}$ ) or $I\left(\mathrm{CO}_{2}\right)>4 \times 2^{13} \mathrm{~W} / \mathrm{cm}^{2}$. Although the calculations used to arrive at these thresholds have not included pump rise times it is evident that this new saturation mechanism can be quite important in moderate intensity or short wavelength laser experiments.

\section{F. Properties of Coupled Modes}

Until now we have focused on mode coupling as a beat wave saturation mechanism. However, the higher $k$ modes which are generated are of interest in their own right because 1) the higher $k$ modes, unlike the high-phase velocity beat wave, can interact strongly with the background electrons, and 2) the generation of an infinite number of higher $k$ modes can lead to quasi-turbulent electrostatic spectrum which is highly undesirable for particle acceleration [2]. 
Although real-life plasmas would ideally be studied for a parameter regime lying somewhere between the two extremes of the warm-plasma and cold-plasma models, a great deal can be inferred about the spectrum of the coupled modes from the cold-plasma treatment. Equations (16a)-(16d) give the temporal evolution of the first few coupled modes as inferred from the total electric field given in (13).

The most distinguishing signature of the electrostatic mode coupling spectrum for cold (and cool) plasmas is the presence of modes which are fundamental in $\omega_{p}$ but harmonic in the ripple wavenumber $k_{i}: \omega \cong \omega_{p 0}, k=k_{p}$ $\pm m k_{i} \cong \pm m k_{i}\left(k_{p} \ll k_{i}\right)$. Although an infinite number of wavenumber harmonics are generated, only a finite number can be expected to be observed experimentally, even in cool plasmas, due to the steady increase of $k \lambda_{d}$ with $m$ for the higher order coupled modes. The relative amplitudes of the various coupled modes can be calculated from (16a)-(16d). However, since (16a)-(16d) are calculated in the cold-plasma limit, estimates of the relative amplitudes can only be approximated for real-life plasmas since these equations do not take into consideration the mismatch between the driver frequency $\left(\omega_{p 0}\right)$ and the Bohm-Gross frequency $\left(\omega_{\mathrm{BG}}\right)$. Qualitatively, (16a)-(16d) and Fig. 2 show that the coupled modes also undergo saturation with the higher order modes saturating at later times and slightly lower amplitudes than the lower order modes. In finite temperature plasmas one can expect these modes to saturate at even earlier times and at lower amplitudes. This has already been seen in the case of the beat wave (cf. (17a) and (25)).

Another important signature of the electrostatic spectrum is its near symmetry in $k$. Equations (13) and (16a)(16d) show explicitly that modes with both plus $\left(\Psi_{+m}\right)$ and minus $\left(\Psi_{-m}\right) k$ 's are excited. For the $m$ th order coupling the respective $k$ 's are $k_{+m}=k_{p}+m k_{i} \cong m k_{i}$ and $k_{-m}=k_{p}-m k_{i} \cong-m k_{i}$ in the underdense plasmas considered here where $k_{p}<k_{i}$. In this case, modes with equal and opposite $k$ 's are expected. This feature will be discussed in greater detail in a later section.

\section{G. Other Coupling Mechanisms}

So far we have considered the beat excitation of electron plasma waves by two laser beams whose frequencies differ by the mean plasma frequency $\omega_{p}$. In the context of the PBWA the two laser beams are assumed to be incident on the plasma from the same direction so that the ponderomotive force of the beating lasers, and therefore the driven plasma wave, have $\vec{k}_{p} \cong \vec{k}_{1}-\vec{k}_{2}$ and consequently $v_{\phi} \cong c$.

In a realistic experimental situation both laser beams can be expected to undergo stimulated Brillouin scattering independently. In such a situation the effective driver consists of not only the beat envelope of the copropagating beams but also the envelope resulting from the beating of one incident laser line with the SBS backscatter from the other line. The latter contribution drives plasma waves with wavenumbers $k_{\mathrm{CPOM}} \cong k_{1}+k_{2}^{\prime}, k_{1}^{\prime}+k_{2} \cong 2 k_{1} \cong$
$2 k_{2}$ where the subscript CPOM denotes "counter propagating optical mixing" [11] and the primes indicate the wavenumbers of the $j$ th pump component $\left(k_{j} \cong k_{j}^{\prime}\right.$ since $\left.\omega_{a c}<<\omega_{1}, \omega_{2}\right)$. The direction of propagation of these large- $k$, low-phase velocity waves can be forward or backward, assuming $\left|\vec{k}_{2}\right|<\left|\vec{k}_{1}\right|$ :

$$
\begin{aligned}
& \vec{k}_{\mathrm{CPOM}}=\vec{k}_{2}-\vec{k}_{1}^{\prime}=\left(k_{2}+k_{1}^{\prime}\right) \hat{x} \cong+\vec{k}_{i} \\
& \vec{k}_{\mathrm{CPOM}}=\vec{k}_{2}^{\prime}-\vec{k}_{1}=-\left(k_{2}^{\prime}+k_{1}\right) \hat{x} \cong-\vec{k}_{i} .
\end{aligned}
$$

The modes excited by CPOM are seen to resemble closely modes generated by mode coupling of the high phase velocity beat wave. This direct generation mechanism for waves with $\omega \cong \omega_{p}$ and $k \cong k_{i}$ leads to ambiguities in the experimentally measured electrostatic spectrum because of the difficulty of resolving the subtle frequency and wavenumber differences between the collinear optical mixing (COM) and CPOM-generated modes.

The previously introduced beat wave excitation model can be modified to facilitate the study of the CPOM problem by replacing the driver in (1) with its CPOM equivalent:

$$
\ddot{E}+\omega_{p}^{2}(x) E+c_{e}^{2} E^{\prime \prime}=\omega_{p 0}^{2} F_{\mathrm{CPOM}} \sin (\Delta k x-\Delta \omega t)
$$

where (for CPOM) $\Delta k= \pm\left[\left|\vec{k}_{1}\right|+\left|\vec{k}_{2}\right|\right] \cong k_{i}, k_{p}=$ $\left|\vec{k}_{1}-\vec{k}_{2}\right|$ as before, and $F_{\mathrm{CPOM}}=\frac{1}{2} \alpha_{i} \alpha_{j}^{\prime}\left[\Delta k / k_{p}\right]$ is the normalized laser intensity modified for the larger $k$ CPOM beat envelope and to take into account the fact that one pump component $\left(\alpha_{j}^{\prime}\right)$ has been fostered by SBS. The analysis following (1) and (2) is general and one can therefore write the CPOM-driven plasma wave electric field in the same form as given in (13) and (16a)-(16d). Noting that for CPOM $\Psi_{m}(\mathrm{CPOM})=\Delta k \pm m k_{i} \cong(m$ $\pm 1) k_{i}=\Psi_{m \pm 1}$ for forward/backward propagating $k=$ $k_{i}$ modes, the CPOM field is

$$
E_{\mathrm{CPOM}}=\sum_{m=-\infty}^{\infty} a_{m} \cos \Psi_{m \pm 1}
$$

where the $a_{m}$ are as given in (16a)-(16d). The CPOM field, unlike the COM field, is seen to be asymmetric in its indices; the $(m+1)$ th coupled mode grows as $a_{m}$. The zero-order or beat wave component is a $k=k_{i}$ wave; the first coupled modes are the $k=0$ and $k=2 k_{i}$ modes; the second coupled modes are the $k=-k_{i}$ and $k=3 k_{i}$ modes and so on.

In an attempt to resolve the ambiguity which arises when both CPOM and COM are possible it is natural to ask to what amplitude the resulting CPOM fields are driven. Equation (23) indicates that the saturation behavior of the $(m+1)$ th coupled mode is governed by the $m$ th temporal coefficient (cf. (16a)-(16d)). For example, assume conditions are such that a forward-propagating $\left(\vec{k}=\vec{k}_{i}\right.$ ) beat wave is excited. The beat excited wave evolves as $a_{0}(t)$ given in (16a) and therefore saturates at an amplitude 


$$
E(\mathrm{CPOM}, \text { sat })=0.735 \frac{2 F_{\mathrm{CPOM}}}{\epsilon} .
$$

Comparing the COM (17b) and CPOM (32) $k=k_{i}$ mode saturation amplitudes one finds that

$$
\frac{E(\mathrm{CPOM}, \mathrm{sat})}{E(\mathrm{COM}, \mathrm{sat})}=\left(\alpha_{j}^{\prime} / \alpha_{j}\right)\left(\Delta k / k_{p}\right) .
$$

For PBWA experiments, where large $\gamma_{\text {phase }}=\omega_{0} / \omega_{p}$ is desirable, we take a typical value of $\omega_{0} / \omega_{p} \cong 10$ or $\Delta k / k_{p}$ $\cong 20$. CPOM can in this example provide a significant or possibly dominant contribution to the experimentally detected levels of $k=k_{i}$ electrostatic modes when

$$
\alpha_{j}^{\prime} / \alpha_{j} \cong\left[I_{\text {SBS }} / I_{\text {incident }}\right]^{1 / 2} \cong 1 / 20
$$

or

$$
I_{\mathrm{SBS}} / I_{\text {incident }} \cong 1 / 4 \text { percent }
$$

a condition easily met in most high-intensity laser experiments. Finer points of the CPOM excitation mechanism will be addressed in a later section.

\section{H. Mode Coupling of Plasma Waves Driven by SRS}

In the context of the PBWA the subject of mode coupling has arisen as an incidental process encountered while attempting to excite large-amplitude high phase velocity electron plasma waves for the purpose of particle acceleration. Although the importance of mode coupling as a beat wave saturation mechanism has been demonstrated, we have thus far said little regarding its more widespread implications and the role it can play in physical situations other than the PBWA.

An example of such a physical situation is that of stimulated Raman scattering (SRS) of incident laser light. In SRS a single-frequency laser beam is incident on an underdense plasma $\left(\omega_{\text {laser }}<\omega_{p} / 2\right)$. For sufficient laser intensity the nonlinear current from the ponderomotive force of the laser acting on thermal density fluctuations $(\tilde{\omega}, \tilde{k})$ emits radiation which beats with the incident radiation (laser) and reinforces the initial density fluctuation. This perturbation grows exponentially in time to form a plasma wave. Note that the frequency of the resulting plasma wave may or may not equal the plasma wave frequency obtained under beat excitation. SRS is usually envisioned to occur in plasmas with sufficiently long density gradient scale lengths so that em-es $\omega$ and $k$ matching is maintained over sufficient distance to generate coherent electromagnetic backscatter. When quasi-static small-scale density fluctuations are present such that $k_{i}<2 \pi / L_{n}, L_{n}$ $=(1 / n(\partial n / \partial x))^{-1}$, mode coupling of the SRS-driven plasma waves can occur resulting in the excitation of modes with $k=k_{\mathrm{SRS}} \pm m k_{i}$. In underdense plasmas, $k_{\mathrm{SRS}}$ $\cong k_{i}\left(\omega_{p}<\omega_{\text {laser }}\right)$ and the driven and coupled modes have wavenumbers given respectively by $k_{i}$ and $(1 \pm$ $m) k_{i}$. Unlike the CPOM case, the possibility of both the $+k_{i}$ and $-k_{i}$ modes exists without two-frequency laser excitation; i.e., when operating the laser on a single line the spectral ambiguity encountered in two-frequency op- eration is not encountered. Detection of a backward propagating electrostatic $k=k_{i}$ mode is conclusive evidence for the mode coupling process.

The analysis of SRS in the presence of a ripple is far less straightforward than for beat excitation. One crude approach is to consider the evolution of the plasma wave from a coherent oscillation to the coupled spectrum. In this approach the work of Kaw et al. [8] is applicable. However, a more realistic approach is to consider how the SRS and ripple (SBS) interdependently evolve together in time. Such a view has been taken by Aldrich et al. [12] who have solved a set of coupled fluid equations for the electromagnetic vector potential and the particle fluctuations at quarter-critical density. When the ion and plasma waves (SBS and SRS) are decoupled mathematically the SRS and SBS instabilities grow independently with wavenumbers $2 k_{0}$ and $3 / 2 k_{0}$, respectively ( $k_{0}=$ pump wavenumber). When the coupling is included, the early time behavior is similar to that in the uncoupled case. However, at later times the plasma wave electric field phase locks to the $2 k_{0}$ ion fluctuation. This represents the first coupling: $3 / 2 k_{0} \rightarrow 7 / 2 k_{0},-1 / 2 k_{0}$. As time progresses the electric field continues to develop spatially. In $k$ space the coupling continues as $3 / 2 k_{0} \rightarrow 7 / 2 k_{0},-1 / 2 k_{0} \rightarrow$ $11 / 2 k_{0},-5 / 2 k_{0} \cdots$. The modes evolve qualitatively much as seen in Fig. 2 and therefore indicate the possibility of saturation of the SRS much as the beat wave saturates due to mode coupling in the presence of an SBS induced ripple. Abrupt quenching of Raman excited plasma waves has been reported by Walsh et al. [14], and Villeneuve $e t$ al. [15] have suggested the possibility of mode coupling playing a role in their experiment.

\section{Mode Coupling in the Presence of SBS Harmonics}

Thus far we have considered density ripples which we have envisioned to arise from SBS-driven ion acoustic waves of wavenumber $k=k_{i}$ where $k_{i} \cong 2 k_{\text {laser }}$. This has been shown to lead to ambiguities in the electrostatic $\omega$, $k$ spectrum when both COM and CPOM occur because of the degeneracy of the $\left(\omega_{p}, k_{i}\right)$ mode (and others). Another complication exists if we consider the role of ion wave harmonics [13]. The CPOM and COM coupled mode spectra now contain the components $(1 \pm m) k_{i}$ and $k_{p} \pm$ $m k_{i}$, respectively. Assuming that the ion wave harmonics cannot exist without the fundamental it can be seen that not only do degeneracies exist between the COM and the CPOM spectra, but also within each of the spectra individually. For example, in COM the mode $\left(\omega_{p}, 2 k_{i}\right)$ can arise from $\left[\omega_{p}, k_{p}+2 \times\left(1 k_{i}\right)\right] \cong\left(\omega_{p}, k_{i}\right)$ or from $\left[\omega_{p}\right.$, $\left.k_{p}+1 \times\left(2 k_{i}\right)\right] \cong\left(\omega_{p}, 2 k_{i}\right)$. Similar examples hold for CPOM spectra.

The amplitudes of the modes resulting from mode coupling of ion harmonics can also be expected to be larger than those resulting from coupling from the fundamental because, from (16a)-(16d), the saturation amplitudes scale as $\epsilon^{-m}$ where $m$ is the harmonic number.

In this context we point out yet another possible process which can lead to the broadening of the $k$ spectrum of the 


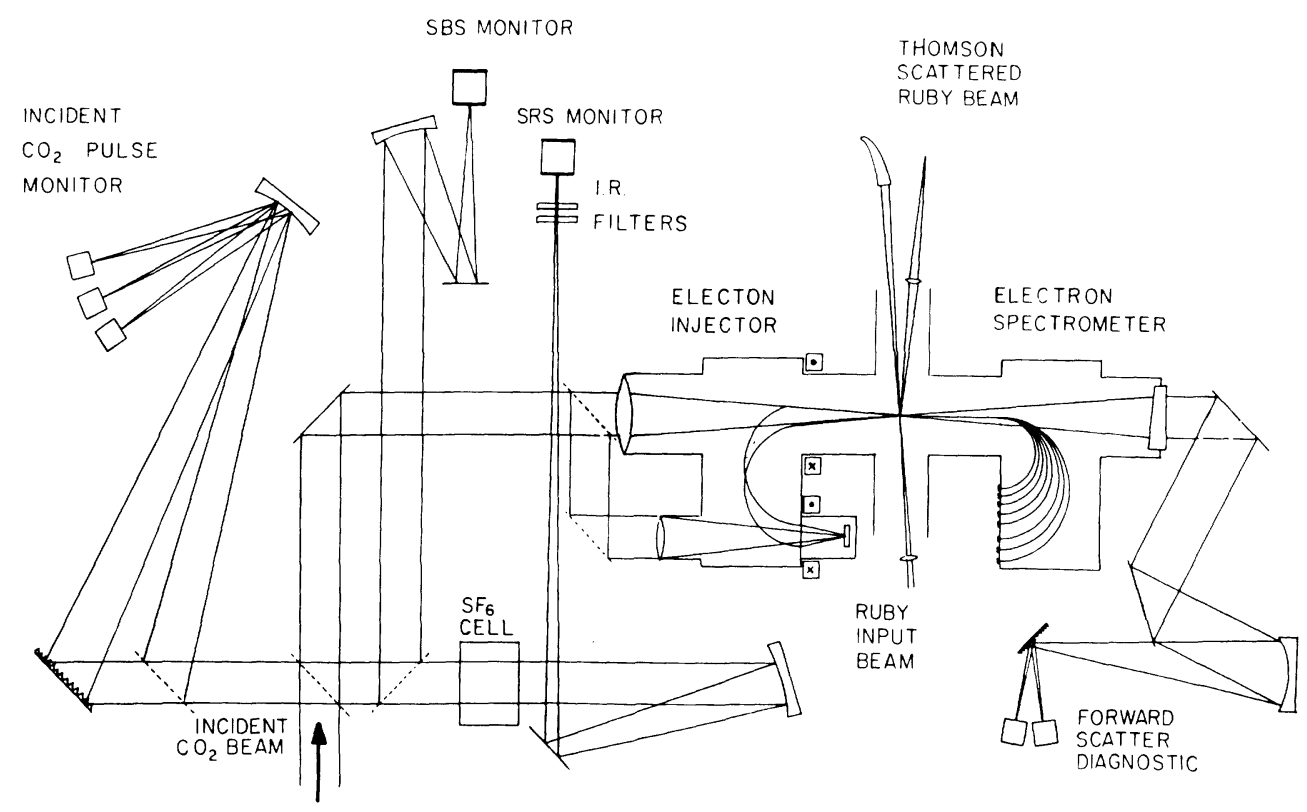

Fig. 4. Diagram of the $\mathrm{CO}_{2}$ laser, ruby laser, vacuum chamber, and diagnostic systems used in the experiments. The electron injector shown in the diagram was not used in the experiments reported on in this paper.

plasma waves with $k \cong k_{i}$ (Raman scatter for $\omega_{p} \ll \omega_{\text {laser }}$, thus $\Psi_{1}$ mode in COM or the $\Psi_{0}$ mode in CPOM). This is the parametric decay of the plasma wave into a backward propagating plasma wave and an ion-acoustic wave: $\left(\omega_{p}, k_{i}\right) \rightarrow\left(\omega_{p}-\omega_{a c},-k_{i}\right)+\left(\omega_{a c}, k_{i}\right)[16]$. This process can be seeded by the second harmonic of the SBS-driven ion wave which has frequency and wavenumber $\left(2 \omega_{a c}\right.$, $2 k_{i}$ ). An interesting aspect of all these coupling processes is that the evolution of the plasma wave takes place in one spatial dimension. Even here the physics is extremely rich. If one allows for angular scattering even more possibilities exist.

\section{Experimental Apparatus and Parameters}

Before giving a detailed description of the various subsystems which comprise the experimental system we will give a brief overview. Fig. 4 shows a diagram of the system and is intended as a reference for the following sections.

The target plasma is first created by a capacitive discharge across the gap between two electrodes in about two torr of an air-hydrogen gas mixture. At some specified time after the plasma discharge is initiated, the $\mathrm{CO}_{2}$ laser oscillator, amplifiers, and diagnostic ruby laser flashlamp are triggered. One pulse, selected from the modelocked $\mathrm{CO}_{2}$ oscillator output train, is amplified to about $10-20 \mathrm{~J}$ and is focused onto the plasma by a lens which serves a second function as a vacuum window on the vacuum vessel. Concurrently, a small portion of the $\mathrm{CO}_{2}$ laser pulse is tapped off and used to trigger a spark gap, which in turn fires the $Q$ switch of the Thomson scatter diagnostic ruby laser. In this way the timing of the ruby laser pulse can be adjusted such that it and the $\mathrm{CO}_{2}$ laser pulse arrive simultaneously at the interaction region within the plasma. As the plasma beat wave is created by the two-frequency
$\mathrm{CO}_{2}$ laser pulse, ruby laser light is scattered at an angle which depends on the plasma wavenumber. The light emerging at this angle is collected by output optics and transferred by a fiber optic to a spectrograph. The spectra are either time integrated and displayed by a 500-channel optical multichannel analyzer (OMA) or time resolved by a streak camera and recorded on film. Back- and forwardscattered $\mathrm{CO}_{2}$ laser light is also analyzed for spectral content and temporal character using either an infrared grating spectrograph or bandpass IR filters in conjunction with a liquid-helium-cooled $\mathrm{Cu}-\mathrm{Ge}$ detector.

\section{A. $\mathrm{CO}_{2}$ Laser System}

The $\mathrm{CO}_{2}$ laser system consists of a TEA oscillator cavity followed by two stages of amplification. The oscillator is actively modelocked by a $1-\mathrm{cm}^{2} \times 1-\mathrm{cm}$ germanium Bragg cell which is placed within the cavity at the beam waist. To facilitate two-frequency operation (for the beat wave experiments) an evacuated cell is situated within the oscillator cavity. For single-line operation and for alignment, when more beam energy is desirable, the cell is left evacuated. Two-frequency operation is accomplished by introducing traces of absorbing gases (Freon 115, 152) into the cell. The output beam, consisting of a train of 2ns pulses, is polarized predominantly in the vertical plane by Brewster windows within the cavity. A spark-gapdriven CdTe Pockels Cell and an analyzer act as a pulse "switchout" for single-pulse operation. The system can also be operated in a "full train" mode by inserting a 1 /4-wave plate after the Pockels cell. This allows all but one pulse to pass providing more oscillator beam energy when needed for system alignment.

The switched-out pulse is then passed through a $2: 1$ Keplerian beam expanding telescope which allows more efficient use of the amplifier gain, provides a means for 
controlling beam divergence, acts as a spatial filter when a pinhole is placed at the focus, and acts as a "plasma shutter" to protect sensitive "upstream" optics from large levels of amplified laser light backscatter. The expanded beam is then double passed through a Lumonics 103 amplifier and amplified to an energy of about $200 \mathrm{~mJ}$.

At this stage the beam is optically stored for about 100 ns to allow sufficient time for buildup of the Thomson scatter ruby laser gain (described below). Following the optical storage leg the beam is double passed through a pair of Lumonics $600-\AA$ amplifiers where it is amplified to about $10 \mathrm{~J}$. The final beam is directed by two copper turning mirrors along the vacuum chamber axis $(z$ axis) where, after passing through an $\mathrm{f} / 7.5 \mathrm{ZnSe}$ lens, it is focused to a point within the interaction region with a focal spot intensity of about $10^{13} \mathrm{~W} / \mathrm{cm}^{2}$.

Prior to entering the focusing optics, the beam passes through a beam splitter which allows us to collect a small portion of both the incident and backscattered laser light (cf. Fig. 4). The incident light was used to monitor pulse height, shape, energy, and spectral content. The backscattered light was used to monitor light which was backscattered by SBS or SRS and, when analyzed for spectral content, provided information on plasma temperature and density, respectively.

To monitor forward-scattered $\mathrm{CO}_{2}$ laser light collection optics were placed along the $z$ axis at the output vacuum port and used to direct the light onto a grating which is mounted on a calibrated rotation stage. The diffracted light was then focused onto a liquid-helium-cooled copperdoped germanium IR detector. This monochromator was calibrated using the various orders of an $\mathrm{HeNe}$ test laser and the 10.6- $\mu \mathrm{m}$ line of the $\mathrm{CO}_{2}$ laser.

\section{B. Collective Thomson Scattering Diagnostic}

Collective Thomson scattering ( $\alpha \geq 6$ ) [17] has been used extensively throughout the experimental work reported on in this paper. The Thomson scattering system consists of the synchronized ruby laser and its associated optics. For an in-depth description of the Thomson scattering system used for the experiments described here, the reader is referred to [18], [19], and [20].

\section{Plasma Preionization}

As mentioned above the $\mathrm{CO}_{2}$ laser excited the plasma beat wave in a preformed target plasma. This plasma is formed by discharging a capacitor across the gap between two electrodes which are fixed within the vacuum vessel. The plasma is taken to full ionization upon arrival of the $\mathrm{CO}_{2}$ laser pulse. Preforming the plasma yields more uniform and reproducible plasmas and expends less laser energy for full ionization than a neutral gas target. The arc discharge is typically created in a 2-torr hydrogen-air gas mixture. Two types of electrodes have been successfully used; a rail-gap electrode $3 \mathrm{~mm} \times 20 \mathrm{~mm}$ and a ball-andpoint electrode. The rail-gap configuration appeared to give a more uniform plasma along $\hat{z}$ but the ball-and-point electrode was used for most of the experiments because it yielded a plasma which was more reliably positioned along the $z$ axis. Both types of electrodes suffer from severe surface erosion of the cathode due to bombardment by energetic ions. It was found that the most controlled method of varying the plasma density (inferred from the frequency shift of the SRS backscatter) was to vary the relative timing of the initiation of the arc discharge and arrival of the $\mathrm{CO}_{2}$ laser pulse. We hypothesize that this was due to thermal expansion of the neutral gas in the partially preionized discharge. By adjusting the arc timing so that the $\mathrm{CO}_{2}$ laser arrives later in the discharge after appreciable neutral gas expansion has occurred, fewer neutrals are available for ionization by the laser and the resulting plasma density is lower. Similarly, early arrival of the $\mathrm{CO}_{2}$ laser pulse as a rule yielded higher plasma densities. The required timing variation of the arc trigger was typically $1-3 \mu \mathrm{s}$ with respect to the $\mathrm{CO}_{2}$ laser trigger.

\section{EXPERIMENTAL Results}

In carrying out our studies of beat excited electron plasma waves several classes of experiments have been performed. We begin with a review of the experiments performed.

In that beat wave excitation is a resonant process, the success of which depends on one's ability to create a resonant density plasma of sufficient length $\left(\cong 10 \lambda_{e s}\right)$, our initial experiments focused primarily on the characterization of the plasma. A most important diagnostic of plasma conditions (density and scale length) was provided by collective Thomson scattering from plasma waves produced by stimulated Raman backscatter.

Having established and adjusted plasma conditions, beat excitation was achieved. Thomson scattering from the beat-excited plasma waves was again critical in verifying resonance, interaction length, and spectral content. The forward-scattered light was analyzed as an independent check to verify the excitation of the high-phase-velocity beat wave.

Further study of the Thomson scatter spectra at various scattering angles led to the discovery of mode coupling in beat wave experiments. As will be discussed, many mode coupling mechanisms are possible in an underdense plasma because of the similarity of the wavenumbers of SBS-driven ion waves and SRS-driven plasma waves.

\section{A. Characterization of the Plasma}

The arc plasma production technique, although used quite successfully in SBS experiments, was the subject of initial testing. Of particular concern was whether or not a plasma of sufficient density $\left(10^{17} \mathrm{~cm}^{-3}\right)$ and length $(10 \lambda$ $\cong 1 \mathrm{~mm}$ ) could be produced.

To answer this question a single pulse of a single-frequency $\mathrm{CO}_{2}$ laser beam was focused onto the preformed arc plasma created in a variety of neutral gases. The backscattered laser light was split into two beams. One was detected directly and used to monitor SBS backscatter. The other was passed through a gas cell $\left(\mathrm{SF}_{6}, 10.6 \mu \mathrm{m}\right.$ absorbing) and a series of interference filters. These filters 


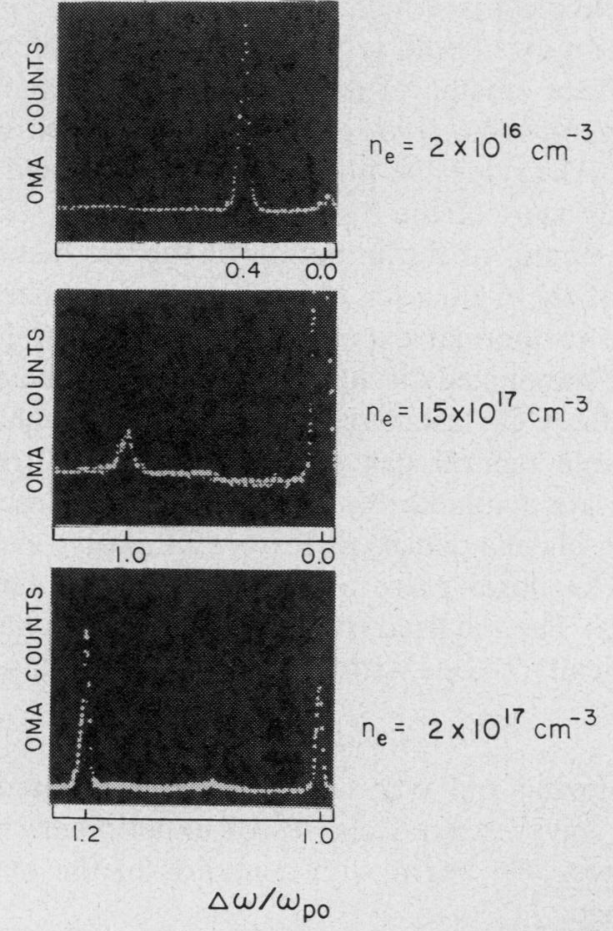

Fig. 5. Thomson scatter frequency spectra of SRS-produced plasma waves. The three different $\omega_{p}$ shifts shown in these three examples correspond to three different plasma densities.

were low- (frequency) pass filters which passed light above 11.1 and $11.75 \mu \mathrm{m}$. Selectively attenuating the 10.6- $\mu \mathrm{m}$ (SBS) component provided sufficient signal-tonoise ratio for detection of the frequency-downshifted (wavelength-upshifted) SRS backscatter. Since the frequency shift of the SRS backscatter equals the plasma wave frequency $\left(\cong \omega_{p}\right)$ at the scatter layer, these two filters cut off at frequencies corresponding to plasma densities of $3 \times 10^{16}$ and $1.2 \times 10^{17} \mathrm{~cm}^{-3}$, respectively. Substitution of filters while observing the SRS backscatter allowed us to estimate roughly the plasma density while varying arc parameters since no low-density SRS backscatter could pass through the $11.75-\mu \mathrm{m}$ filter. A 10.6$\mu \mathrm{m}$ bandpass filter $(10.6 \pm 0.2 \mu \mathrm{m})$ was substituted for the low-pass filters. The absense of a signal when the bandpass filter was in place verified that the signals were indeed frequency shifted and not due to stray light.

SRS measurements were carried out using several neutral gases and mixes. The gas mix which produced the most reproducible Raman backscatter consisted of 1 torr each of hydrogen and air, although on various occasions, significant variations of this mix were successfully used (e.g., 1 torr $\mathrm{H}_{2}, 0.1$ torr air). The plasma density, inferred from the frequency shift of the backscatter, could be roughly tuned to resonance by adjusting the arc timing and voltage. Fig. 5 shows Thomson scatter spectra obtained from scattering from SRS-driven electron plasma waves at three different densities. With the gas mix with which we typically operated (1-torr $\mathrm{H}_{2}, 1$-torr air) densities of $2 \times 10^{16}-2 \times 10^{17} \mathrm{~cm}^{-3}$ were attainable.

A crude estimate of plasma uniformity was obtained

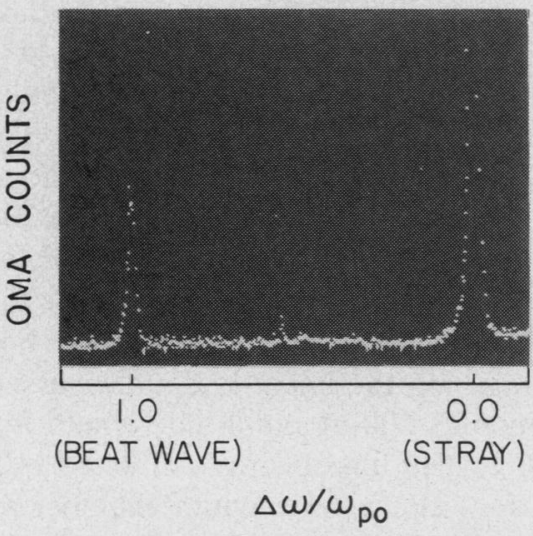

Fig. 6. Thomson scatter frequency spectrum of the high phase velocity beat-excited plasma wave (beat wave).

from the gradient threshold for the SRS instability. Since SRS is observed, the gradient threshold is assumed to have been exceeded and the plasma length is inferred to be greater than or equal to the scale length appearing in the gradient threshold formula [21] $\left(v_{\text {osc }} / c\right)^{2}=2\left(k_{\text {laser }} L_{n}\right)^{-1}$. Using our laser intensity of $10^{13} \mathrm{~W} / \mathrm{cm}^{2}$, we estimate $L_{\text {plasma }}>8 \mathrm{~mm}$.

\section{B. Excitation of the Beat Wave}

The ability to control the plasma conditions, as demonstrated by the SRS measurements, provided us with a well-controlled environment in which to conduct a study of plasma beat wave excitation.

The most direct evidence for existence of the plasma beat wave was obtained from small-angle Thomson scatter spectra. In these experiments the diagnostic ruby laser optical system, as described previously, was aligned for a scatter angle of $0.4^{\circ}$ ( $\omega$ and $k$ matching to the $\lambda \cong 100$ $\mu \mathrm{m}$ beat wave). The $\mathrm{CO}_{2}$ pump laser was initially operated on a single laser line $(10.59 \mu \mathrm{m})$. Arc parameters were varied until the SRS backscatter frequency shift measurements indicated that a resonant plasma density had been attained. The $\mathrm{CO}_{2}$ laser was then operated on two frequencies ( 10.59 and $9.56 \mu \mathrm{m}$ ) by introducing absorbing gases into the $\mathrm{CO}_{2}$ laser cavity gas cell.

Fig. 6 shows a Thomson scatter frequency spectrum taken at $0.4^{\circ}$. As expected, the frequency shift of the beat wave spectral peak (left) corresponds exactly to the frequency separation of the two laser lines. The peak on the right, unlike the SBS spectral peak appearing in the $2 k_{\mathrm{CO}_{2}}\left(7 \frac{1}{2}^{\circ}\right)$ Thomson scatter spectra of Fig. 5 , is due to stray ruby laser light (i.e., the $2 k_{\mathrm{CO}_{2}}$ SBS waves are not $\omega$ and $k$ matched to the ruby laser at $0.4^{\circ}$ ).

Further evidence for the existence of the plasma beat wave was obtained by analyzing the transmitted (forwardscattered) $\mathrm{CO}_{2}$ laser light. Fig. 7 schematically shows the transmitted $\mathrm{CO}_{2}$ laser light spectrum. In addition to the two $\mathrm{CO}_{2}$ laser lines at 10.6 and $9.6 \mu \mathrm{m}$ two discrete lines frequency downshifted from the $10.6-\mu \mathrm{m}$ laser line by $\omega_{p 0}$ and one line frequency upshifted from the 9.6- $\mu \mathrm{m}$ laser line by $\omega_{p 0}$ were detected. These "Stokes" (frequency- 


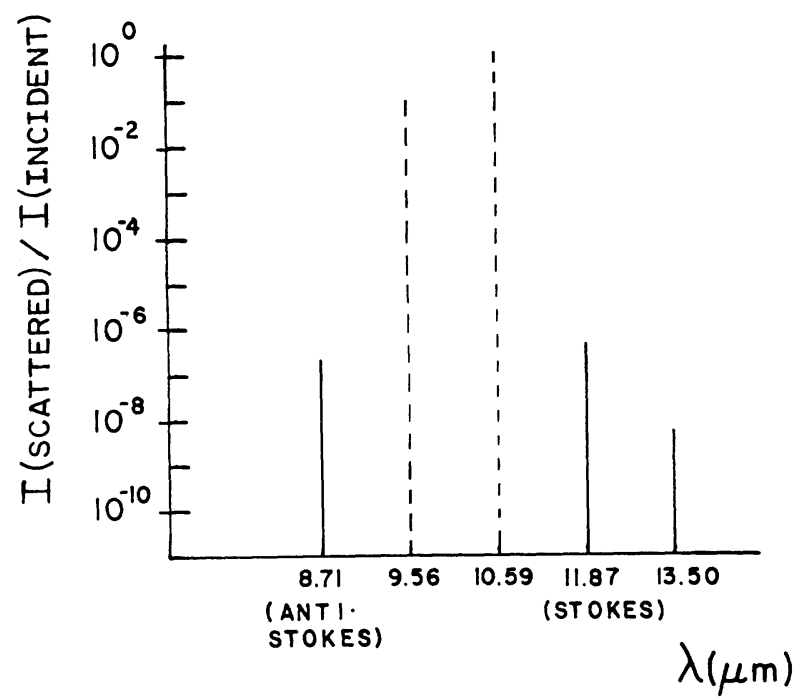

Fig. 7. Schematic representation of the measured forward-scattered (transmitted) $\mathrm{CO}_{2}$ laser light frequency spectrum. The dashed lines correspond to the two (10.6 and $9.6 \mu \mathrm{m}) \mathrm{CO}_{2}$ laser lines.

downshifted) and "anti-Stokes" [22]-[24] (frequencyupshifted) lines are attributed to scattering of the $\mathrm{CO}_{2}$ laser light by the plasma beat wave. Note also that, in principle, contributions to the first Stokes line and the first antiStokes line can arise from scattering of the 9.6- and 10.6$\mu \mathrm{m}$ lines from the second harmonic of the beat wave. Similarly, the second Stokes and second anti-Stokes can scatter off of the beat wave fundamental and make contributions to the first Stokes and first anti-Stokes lines.

This situation is further complicated by the fact that the forward-scatter measurements integrate along the entire axial extent of the interaction region where, as one might expect, the spectral character of the cascaded electromagnetic spectrum varies along the direction of propagation of the plasma wave. It is therefore possible to draw only limited conclusions from the relative amplitudes of the various spectral features in Fig. 7. However, the structure of the electromagnetic spectrum (spectral peaks spaced with $\Delta \omega=\omega_{p 0}$ ) is unambiguous evidence for the existence of the high phase velocity beat wave since only the short- $k$ beat wave can $\omega$ and $k$ match to the $\mathrm{CO}_{2}$ laser lines to produce the observed electromagnetic spectrum. As expected, when the $\mathrm{CO}_{2}$ laser was operated on a single laser line, no Stokes or anti-Stokes lines were observed. Also, when the laser was operated on two frequencies but with vacuum in place of the plasma (null test), no Stokes or anti-Stokes sidebands were observed.

Important to the coherence of the plasma beat wave is the discreteness in $\omega$ and $k$ of that mode. Discreteness in $\omega$ has already been demonstrated in the Thomson scatter spectrum of Fig. 6. A detailed plasma beat wave $k$ spectrum, shown in Fig. 8, was taken by scanning the fiber optic in the ruby laser Thomson scatter detection plane. This spectrum was taken over many shots and statistically found to peak at the expected $k_{p}=k_{10.6}-k_{9.6}$ with a halfpower width of about 20 percent (of $k_{p}$ ). Note also that the discreteness in $k$ of the transmitted $\mathrm{CO}_{2}$ laser light

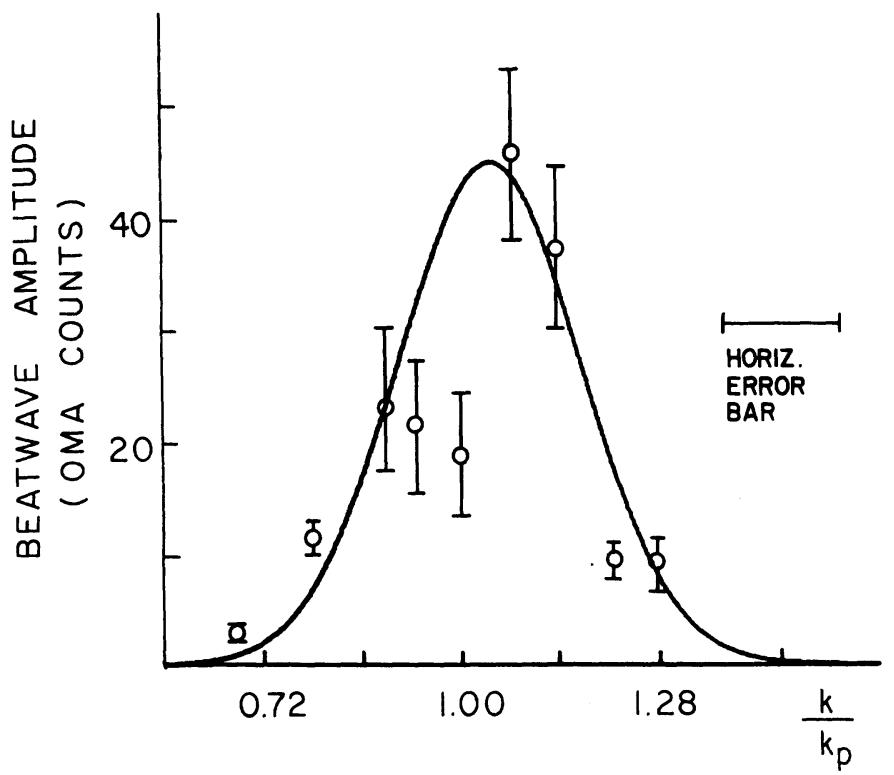

Fig. 8. Wavenumber spectrum of the high phase velocity, beat-excited plasma wave obtained by scanning the Thomson scatter detection fiber in the $\hat{z}$ direction. The horizontal error bar shown is due to the positioning error and finite size of the light collecting fiber optic. The solid curve is intended to serve as a visual reference only.

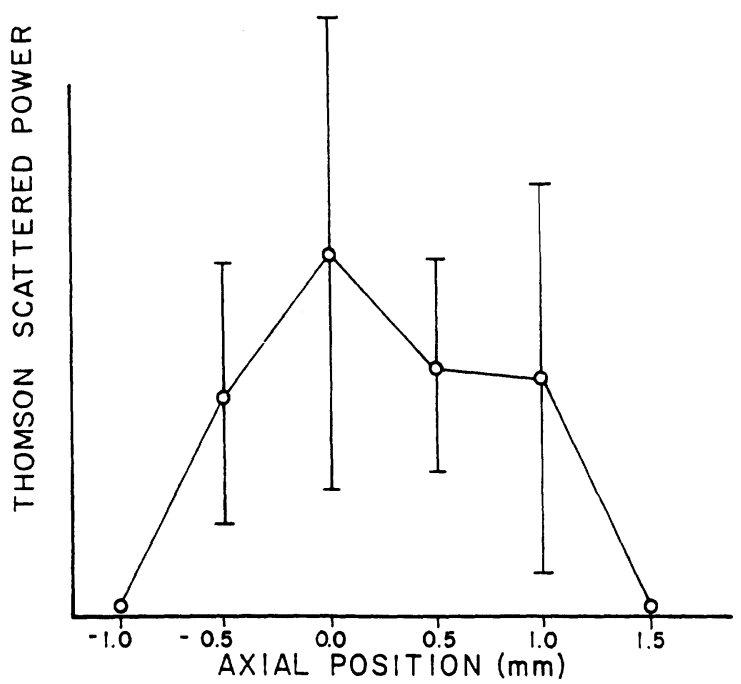

Fig. 9. Axial profile of the beat wave inferred from Thomson scatter measurements (cf. Fig. 12).

supports the conclusion that the plasma beat wave is discrete. As mentioned above, when the fiber was scanned to a position corresponding to the angle for scattering from the plasma beat wave second harmonic, no signal was observed indicating that the beat wave second harmonic was down in amplitude by a factor of 10 or greater.

A method to estimate the plasma interaction length has been presented above for the nonresonant (single-frequency) excitation. The cylindrical Thomson scatter optical system provided us with a method with which to measure directly the resonant interaction length of the beat wave. This technique is described in detail in [18]. The results of this measurement are shown in Fig. 9. The interaction region is found to be about $2 \mathrm{~mm}$ or 20 plasma wavelengths in length. Because the plasma wave group 
velocity to phase velocity ratio is so small $\left(v_{g} / v_{\phi} \cong\right.$ $v_{e}^{2} / c^{2}$ ), one can expect that the wave field will grow to its saturation value long before convecting out of the resonant interaction region.

This conclusion is supported by the estimated wavefield amplitude as inferred from the plasma beat wave Thomson scatter measurements. The interaction length and Thomson scatter intensity can be substituted into the Bragg scattering formula [25]

$$
\frac{I_{\text {scat }}}{I_{\text {inc }}}=\frac{L_{p}}{L_{r}} \frac{\tau_{p}}{\tau_{r}}\left[\frac{\pi}{2} \frac{n_{1}}{n_{0}} \frac{n_{0}}{n_{c}} \frac{d}{\lambda_{r}}\right]^{2}
$$

where $L_{p}\left(L_{r}\right)$ is the plasma wave (ruby focus) length, $\tau_{p}\left(\tau_{r}\right)$ is the plasma wave (ruby laser) duration, $d$ is the plasma wave width (perpendicular to $\vec{k}_{e s}$ ), $\lambda_{r}$ is the ruby laser wavelength, $\tilde{n}, n_{0}$, and $n_{c}$ are the perturbation, background plasma, and $\mathrm{CO}_{2}$ laser critical densities, respectively. Based on a maximum measured $I_{\text {scat }} / I_{\text {inc }}$ of $\cong 10^{-10}$ for the ruby probe beam at $0.4^{\circ},(35)$ yields a plasma beat wavefield amplitude of $\cong 1-3$ percent. In arriving at this figure we have included the statistical variation of the scattered probe beam. However, an important effect involving $\omega$ and $k$ matching of the ruby laser probe beam and the beat wave was pointed out by Martin et al. [26], [19]. When this effect is included one is led to estimate the field of the axial component of the beat wave to be a few times larger than the 1-3 percent measured. A detailed discussion of the plasma beat wavefield saturation amplitude will be given below. However, it is worth noting at this point that the measured field amplitude is consistent with the theoretical predictions of Section II.

\section{Observations of Coupled Modes}

To further characterize the electrostatic $\omega-k$ spectrum, Thomson scatter measurements were made at several scatter angles other than $0.4^{\circ}$. These angles, $7 \frac{1}{2}^{\circ}, 15^{\circ}$, and $222^{\circ}$, were chosen so as to achieve $\omega$ and $k$ matching between the ruby laser probe beam and the $2 k_{\mathrm{CO}_{2}}, 4 k_{\mathrm{CO}_{2}}$, and $6 k_{\mathrm{CO}_{2}}$ electrostatic modes, respectively. This allowed us to construct the electrostatic $\omega-k$ spectrum by obtaining the $\omega$ spectrum at the electrostatic $k$ 's of interest.

The results of measurements made at $7 \frac{1}{2}^{\circ}$ using a single $\mathrm{CO}_{2}$ laser line have already been discussed in a previous section. Fig. 10 shows a composite Thomson scatter frequency spectrum taken at $7 \frac{1}{2}^{\circ}$ with a two-frequency $\mathrm{CO}_{2}$ laser beam. The central spectral peak is due to scattering from $k=k_{i}=2 k_{\mathrm{CO}_{2}}$ SBS-driven ion acoustic waves, and is present even with a single-frequency $\mathrm{CO}_{2}$ laser beam. Note that the SBS spectral peak appears to occur at the ruby laser frequency because the small acoustic frequency shift $\left(\omega_{a c} / \omega_{p} \cong 3.5 \times 10^{-3}\right)$ is not resolvable on this scale. The peak on the left is blue shifted from the SBS spectral peak by exactly the $\mathrm{CO}_{2}$ laser line frequency difference. The spectral peak on the right is red shifted by the same amount. The blue- and red-shifted lines correspond to forward $(+\hat{z})$ and backward $(-\hat{z})$ propagating modes in the plasma with $k \cong k_{i}$ (from now on we use $k_{i}$

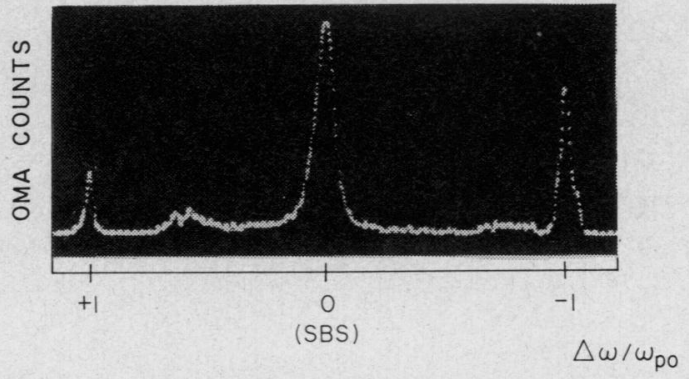

Fig. 10. Composite Thomson scatter frequency spectrum taken at a scatter angle of $7.5^{\circ}\left(k_{e s}=k_{i}\right)$ showing an SBS spectral peak as well as peaks red and blue shifted by the beat frequency $\omega_{p 0}$.

to denote the wavenumber of electrostatic waves with $k$ $\cong 2 k_{\mathrm{CO}_{2}}$ ). Note that for large-angle Thomson scattering one does not encounter $\omega$ and $k$ matching difficulties to the same extent as in the case of small-angle scattering. Because $k_{e s}$ is large $\omega$ and $k$ matching is easily achieved for a nearly normally incident Thomson scatter probe beam and it is the axially propagating component which is indeed probed. One is tempted to attribute the blueshifted line to a $k \cong k_{i}$ Raman-driven plasma wave, whereas no such explanation for the red line is readily obvious. A more plausible explanation is provided by the mode coupling model of Section II which predicts a symmetry in $k$ of the resulting electrostatic (Thomson scatter) spectrum as seen in Fig. 10; that is, both red- and blueshifted lines are expected because modes with $\pm \vec{k}_{i}$ are excited when the high phase velocity plasma beat wave scatters from a density ripple with $k_{\text {ripple }} \cong k_{i}$. Thomson scatter measurements at $7 \frac{1}{2}^{\circ}$ verify the presence of the ripple with $k \cong k_{i}$ driven by SBS from either of the two $\mathrm{CO}_{2}$ laser lines.

Further evidence for the mode coupling process has been obtained while making Thomson scatter measurements at $15^{\circ}$ and $22 \frac{1}{2}^{\circ}$, the angles corresponding to scatter from $k=2 k_{i}$ and $k=3 k_{i}$ modes, respectively. Fig. 11(a) shows a Thomson scatter frequency spectrum taken at $15^{\circ}$. The central peak, due to scatter from the second harmonic of SBS-driven ion waves, is still unresolvable and appears unshifted on this frequency scale. The spectral peaks which are blue- and red-shifted by $\omega_{p 0}$ are due to scatter from forward- $\left(\omega_{p 0},+\vec{k}_{i}\right)$ and backward- $\left(\omega_{p 0}\right.$, $\left.-\vec{k}_{i}\right)$ propagating coupled modes. These modes are generated when the beat wave scatters off of the density ripple: $\left(\omega_{p 0}, \vec{k}_{p}\right)+\left(\omega_{i}, \vec{k}_{i}\right) \stackrel{m=+2}{\longrightarrow}\left(\omega_{p 0},+2 \vec{k}_{i}\right)$ and $\left(\omega_{p 0}\right.$, $\left.\vec{k}_{p}\right)+\left(\omega_{i}, \vec{k}_{i}\right) \stackrel{m=-2}{\longrightarrow}\left(\omega_{p 0},-2 \vec{k}_{i}\right)$. The spectral peaks which are blue and red shifted by $2 \omega_{p}$ are due to Thomson scatter from the $\left(2 \omega_{p 0}, \pm 2 \vec{k}_{i}\right)$ modes which are believed to arise when the beat wave second harmonic scatters from the ion wave ripple: $\left(2 \omega_{p 0}, 2 \vec{k}_{p}\right)+\left(\omega_{i}, \vec{k}_{i}\right) \stackrel{m=+2}{\longrightarrow}\left(2 \omega_{p 0}\right.$, $\left.2 \vec{k}_{i}\right)$ and $\left(2 \omega_{p 0}, 2 \vec{k}_{p}\right)+\left(\omega_{i}, \vec{k}_{i}\right) \stackrel{m=-2}{\longrightarrow}\left(2 \omega_{p 0},-2 \vec{k}_{i}\right)$. The experimental manifestations of the degeneracies of the $\left(n \omega_{p 0}, \pm m \vec{k}_{i}\right)$ modes mentioned in Section II are discussed in greater detail in a later section when other coupling mechanisms are considered. 


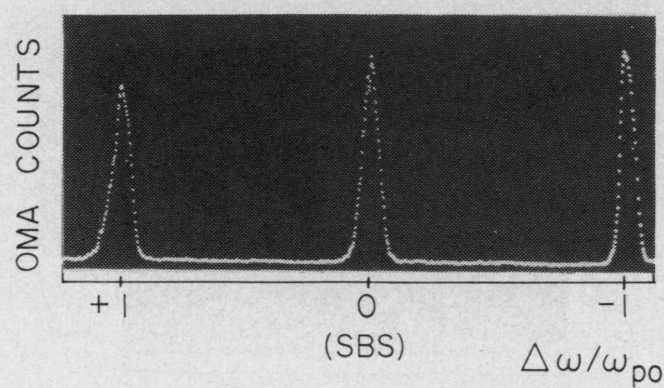

(a)

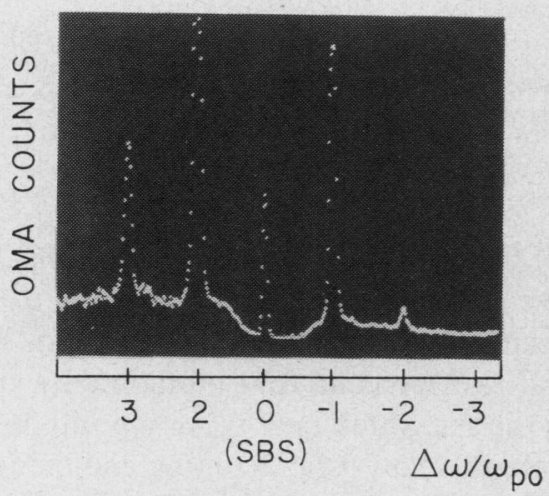

(b)

Fig. 11. (a) Composite Thomson scatter frequency spectrum taken at a scatter angle of $15^{\circ}\left(k_{e s}=2 k_{i}\right)$. The central spectral peak is due to scatter from the second harmonic of the SBS-driven ion-acoustic wave. The peaks red and blue shifted by $\omega_{p 0}$ are due to coupled modes. (b) Thomson scatter frequency spectrum taken at a scatter angle of $22.5^{\circ}$ $\left(k_{e s}=3 k_{i}\right)$ for a single shot of the laser system. The central peak is due to scatter from the third harmonic of the SBS-driven ion-acoustic wave. The modes which are red and blue shifted by $\omega_{p 0}$ and $2 \omega_{p 0}$ are attributed to coupled modes.

Fig. 11(b) shows a $3 k_{i}$ Thomson scatter spectrum taken on a single shot at $22 \frac{1}{2}^{\circ}$ (a lower dispersion grating was used in the Thomson scatter spectrometer and allowed us to record up to the $\pm 3 \omega_{p 0}$ lines simultaneously thereby avoiding the need to assemble composite spectra as in Figs. 10 and 11(a) which were composed of different portions of the Thomson scatter spectrum recorded on different shots). In Fig. 11(b) one again observes an unshifted spectral peak at the ruby laser frequency (center peak) due to scatter from the third ion wave harmonic. The spectral peaks which are red and blue shifted by $\omega_{p 0}$ are due to scatter from the coupled modes ( $m= \pm 3$ coupling to the ion wave). Similarly, the spectral peaks which are red and blue shifted by $2 \omega_{p 0}$ are due to scatter from coupled modes arising when the second beat wave harmonic undergoes an $m= \pm 3$ coupling to the density ripple. Thus far no $3 \omega_{p 0}, \pm 3 \vec{k}_{i}$ modes have been observed. This is believed to be due to the relative weakness of the beat wave third harmonic.

The $\omega_{p 0}, \pm 2 k_{i}$ and $\omega_{p 0}, \pm 3 k_{i}$ modes described above are adequately explained by the mode coupling model of Section II. However, the $2 \omega_{p 0},+3 k_{i}$ lines do not fit neatly into this formalism without the introduction of beat wave harmonics. Although no second beat wave harmonic was directly observed, given the sensitivity of the $0.4^{\circ}$ Thomson scatter system and the characteristics of the forward

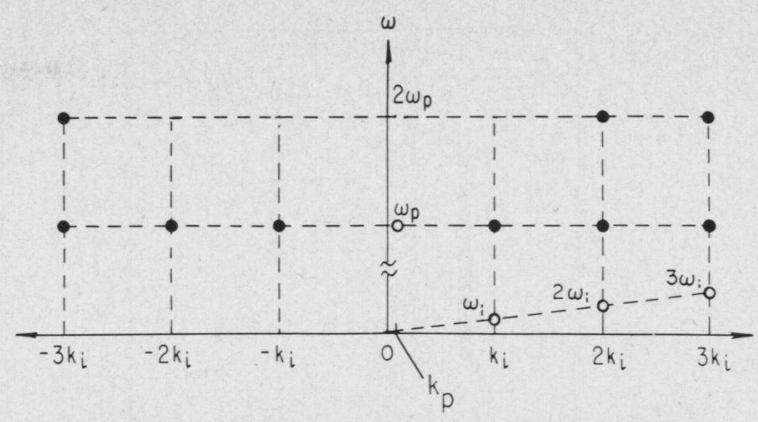

Fig. 12. Summary of the electrostatic modes which have thus far been observed in our experiments. Shown are the beat wave and SBS ion wave harmonics (open dots) as well as those modes exhibiting the signature of coupled modes (fundamental in $\omega_{p 0}$, harmonic in $k_{i}$, solid dots).

electromagnetic scatter spectrum, the possibility of the existence of a beat wave second harmonic remains and these lines are attributed to $m= \pm 2$ and $m= \pm 3$ couplings of the beat wave harmonic through the SBS ion wave $\omega_{i} \cong 0, k_{i}$. Note also that the $\omega_{p 0}, \pm 2 k_{i}$ and $2 \omega_{p 0}$, $\pm 3 k_{i}$ lines could also arise from an $m=1$ coupling of the beat wave through the second and third ion wave harmonics $2 \omega_{i}, 2 k_{i}$ and $3 \omega_{i}, 3 k_{i}$. Fig. 12 summarizes the modes which have thus far been observed at the various Thomson scatter angles. Other possible excitation mechanisms for modes observed at $k_{i}, 2 k_{i}$, and $3 k_{i}$ will be discussed in a later section.

An important criterion for the existence of coupled modes is the simultaneity of the ripple (due to SBS) and the coupled mode. Fig. 13 shows a time-resolved Thomson scatter frequency spectrum taken at $7 \frac{1}{2}^{\circ}$. The SBS ripple and $\omega_{p}, k_{i}$ mode (blue shifted) are seen to coexist in time. Note, however, that the SBS ripple turns on earlier and turns off later than the $\omega_{p}, k_{i}$ mode. This is believed to occur because the SBS is a nonresonant process and has a lower threshold and begins to grow exponentially early in the $\mathrm{CO}_{2}$ laser pulse. Excitation of the beat wave on the other hand is a resonant process and can proceed only after the plasma is brought to the fully ionized resonant density by the $\mathrm{CO}_{2}$ laser. Once the resonant plasma density is finally reached the plasma beat wave, and therefore the coupled mode, are constrained to grow at the growth rate given in Section II, $\gamma=\frac{1}{4} \alpha_{1} \alpha_{2} \omega_{p 0}$. Note also that, due to finite laser pulse rise time, the growth rate early in the laser pulse is lower than at the peak of the pulse since the $\alpha$ 's are functions of time. This effect delays the growth of the beat wave even further.

\section{Estimation of Mode Field Saturation Amplitudes}

The theory of Section II provides a model with which to predict the field amplitude at saturation of the various coupled modes and the beat wave. Experimental estimates of the mode field amplitudes can be obtained from (35) where we assume the same mode spatial profile (i.e., $L_{p}$ ) as was directly measured for the beat wave. However, some subleties in the application of this formula to the two Thomson scatter geometries used in our experiments warnanı further consideration. 


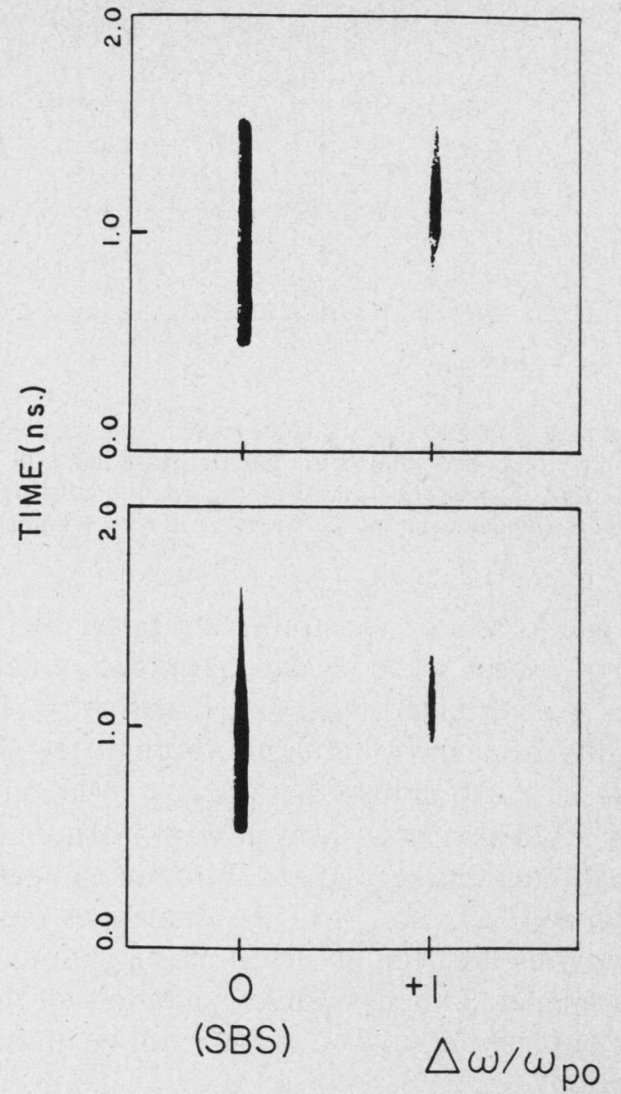

Fig. 13. Two examples of the temporally resolved Thomson scatter frequency spectrum taken at $7.5^{\circ}\left(k_{\text {es }}=k_{i}\right)$ with two frequency laser excitations taken on two separate occasions. The SBS and coupled mode are seen to coexist in time as expected.

\begin{tabular}{|l|c|c|}
\hline & $E\left(\Psi_{0}\right)$ & $E\left(\Psi_{1}\right)$ \\
\hline EXPERIMENT & $3-9 \%$ & $.09-0.9 \%$ \\
\hline $\begin{array}{l}\text { RELATIVISTIC } \\
\text { DETUNING }\end{array}$ & $8 \%$ & - \\
\hline $\begin{array}{l}\text { COLD PLASMA } \\
\text { MODE COUPLING }\end{array}$ & $0.6 \%$ & $0.5 \%$ \\
\hline $\begin{array}{l}\text { WARM PLASMA } \\
\text { MODE COUPLING }\end{array}$ & $3 \%$ & $0.8 \%$ \\
\hline
\end{tabular}

Fig. 14. Summary of the theoretically predicted and experimentally estimated electrostatic field amplitudes for the beat wave $\left(\Psi_{0}\right)$ and first coupled mode $\left(\Psi_{ \pm 1}\right)$ for $p \cong 2$ and $\epsilon \cong 0.04$ as in the experiment.

Equation (35) relates the fraction of scattered Thomson scatter probe beam power to the mode strength measured in $\tilde{n} / n_{0}$. This formula takes into consideration both the spatial and temporal coincidence of the mode of interest and the probe beam [27]. In Fig. 14 we compare the predicted and observed electrostatic field amplitudes. The theoretically predicted amplitudes follow from the saturation values calculated above for warm and cold plasmas (17) and (25) where for warm plasmas we have taken $p$ $=2$ and $\alpha_{1} \alpha_{2}=3 \times 10^{-4}$ as in the experiment. For reference we also compare the predicted beat wave saturation amplitude for relativistic detuning. The measured

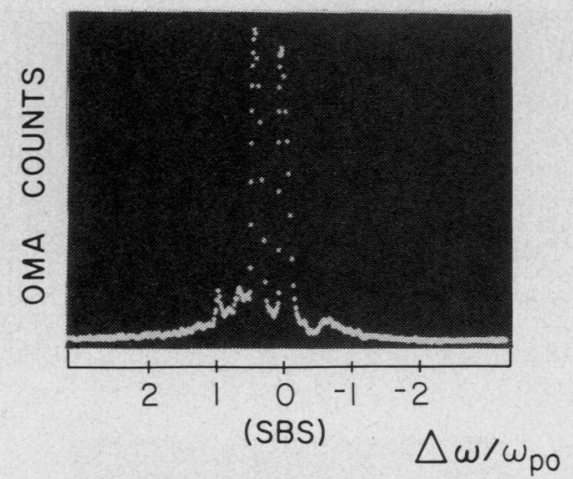

Fig. 15. Thomson scatter frequency spectrum taken at $7.5^{\circ}\left(k_{e s}=k_{i}\right)$ for two-frequency excitation showing peaks shifted by $0.38 \omega_{p 0}$ and 0.69 $\omega_{p 0}$.

amplitude of the beat wave is $\cong 9 \pm 6$ percent. From (25) and (27a) we expect a saturation amplitude of $\cong 13$ percent ( 8 percent including pump rise time) for relativistic detuning and $\cong 3$ percent for saturation by mode coupling. Thus the measured beat wave amplitude is consistent with both the relativistic detuning and mode coupling saturation models. However, observations of the coupled modes and the fair agreement between their theoretically predicted and experimentally measured amplitudes are strongly suggestive of the importance of the contribution of the mode coupling saturation mechanism in our experiment.

\section{E. Other Features of the Electrostatic Spectrum}

In this section we discuss a number of interesting results obtained from Thomson scatter studies of the electrostatic mode spectrum. Although these results often complicate the interpretation of the physics of the experiment, they serve to illustrate the importance of the interrelations of the various physical processes occurring in the plasma.

Under two-frequency excitation the nature of the electrostatic spectrum has been investigated for several electrostatic $k$ 's as described above. The spectra presented there are in good agreement with the mode coupling model of Section II. However, Fig. 15 shows an example of a $2 k_{\mathrm{CO}_{2}} \cong k_{i}$ Thomson scatter frequency spectrum obtained under two-frequency excitation which, although partially explained by the mode coupling model, contains additional spectral features. The central peak is again due to Thomson scatter from the SBS-produced density ripple. The peak on the left is blue shifted from the zero frequency SBS spectral peak by the resonant plasma frequency $\omega_{p 0}\left(\Delta \omega_{\text {lasers }}=\omega_{p 0}\right)$ and is believed to be an $\omega_{p 0}$, $k_{i}$ coupled mode. Also seen are a large spectral peak blue shifted from the zero frequency peak by about $0.38 \omega_{p 0}$ and a small spectral peak blue shifted by $0.68 \omega_{p 0}$. These modes are believed to arise due to the nonresonant excitation of low-frequency $k=k_{i}$ electron plasma waves by SRS at points along the axis within the $\mathrm{CO}_{2}$ depth of focus where the plasma density is different from the beat wave resonant density. 


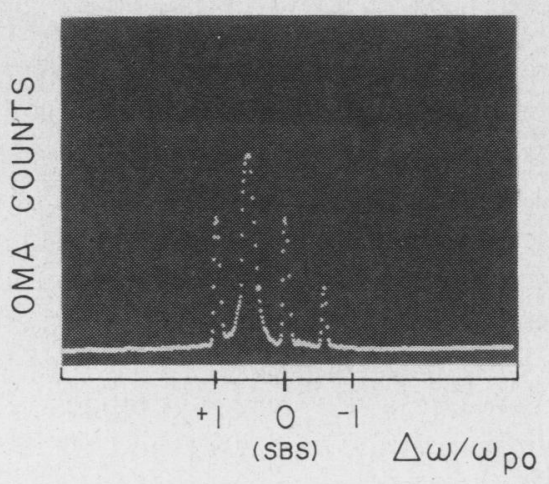

Fig. 16. Thomson scatter frequency spectrum taken at $7.5^{\circ}\left(k_{e s}=k_{i}\right)$ for two-frequency excitation. Clearly seen are spectral features shifted by exactly $\omega_{p 0}$ and $0.5 \omega_{p 0}$.

Fig. 16 shows another case where the low-density $k=$ $k_{i}$ mode occurs with a frequency exactly equal to $\frac{1}{2} \omega_{p 0}$. This is a particularly interesting case because, under these conditions, it is possible to parametrically excite an unstable mode with $\Delta \omega_{\text {lasers }}=2 \omega_{\text {es }} \cong 2 \omega_{p}$ and $\Delta k_{\text {lasers }}=2 k_{\text {es }}$ where $\omega_{p}$ is the plasma frequency of the subresonant density plasma (i.e., $\omega_{p}=\frac{1}{2} \omega_{p 0}$ ) [6]. This mode is similar in character to the resonantly excited beat wave in that it too has $v_{\phi}=\omega_{p} / k_{\text {es }} \cong c$. While the low-frequency $\left(\frac{1}{2} \omega_{p 0}\right)$ mode in Fig. 16 could be due to a low-density SRS-driven plasma wave, it is interesting to speculate on the possibility of this mode arising from mode coupling of the parametrically excited $\frac{1}{2} \omega_{p 0}, \vec{k}_{i}$ mode. Note also the presence of its red-shifted complement, $\frac{1}{2} \omega_{p 0},-\vec{k}_{i}$. Unfortunately, the Thomson scatter measurements used to obtain the $k$ spectrum of the beat wave (Fig. 8) were not extended to include observations at $\frac{1}{2} k_{p}$ where one would expect to observe the $\frac{1}{2} \omega_{p 0}, \frac{1}{2} k_{p}$ mode directly, and we therefore have no conclusive evidence for the existence of the $\frac{1}{2} \omega_{p 0}, \frac{1}{2} k_{p}$ mode itself.

Assuming the blue-shifted $\frac{1}{2} \omega_{p 0}$ mode is the quarter-resonant density, parametrically excited electron plasma wave it is not unreasonable to assume that the blue-shifted $\omega_{p 0}$ mode can arise from mode coupling of the second harmonic of the $\frac{1}{2} \omega_{p 0}, \frac{1}{2} k_{p}$ mode. Note in Fig. 16 that the relatively large amplitude of the $\frac{1}{2} \omega_{p 0}$ spectral peak compared with the $\omega_{p 0}$ spectral peak tends to support this assumption since one expects the coupled mode amplitudes to reflect the relative amplitudes of the fundamental and second harmonic parent mode amplitudes (cf. (16b)). It is also interesting that a mode identical in character (same $\omega$ and $k$ ) to the resonantly excited beat wave can be excited in a nonresonant density plasma; that is, the beat wave fundamental is the same as the second harmonic of the low-frequency parametrically excited mode. The absense of the $\frac{1}{2} \omega_{p 0}$ mode at $2 k_{i}$ and $3 k_{i}$ (i.e., higher order mode couplings of the low-frequency mode) may be due to the enhanced thermal quenching of the coupling in the quarter-resonant density plasma where $k_{i} \lambda_{d}$ is twice as large as in the resonant density case (cf. Section II).

Perhaps the experimental evidence most supportive of the viability of the mode coupling mechanism was ob-

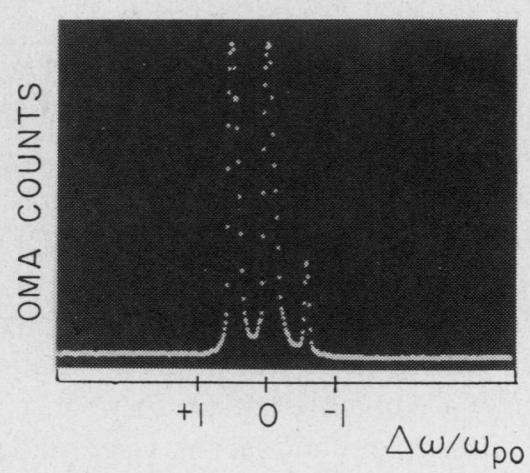

Fig. 17. Thomson scatter frequency spectrum taken at $7.5^{\circ}\left(k_{e s}=k_{i}\right)$ for single-frequency laser excitation. Both forward- and backward-propagating modes symmetrically shifted from the central spectral peak (SBS) by $\omega_{p}$ are clearly observed and attributed to coupled modes driven by SRS.

tained under single-frequency laser pumping of the plasma. As discussed in Section I, under two-frequency pumping many ambiguities exist in the Thomson scatter spectrum because of the similarity of the SBS, SRS, and CPOM wavenumbers $\left(k_{\mathrm{SRS}} \cong k_{\mathrm{SBS}} \cong k_{\mathrm{CPOM}}\right)$. Under single-frequency pumping the CPOM process is not possible and the red-shifted mode $\omega_{p}, m \vec{k}_{i}$ can only arise from mode coupling. Fig. 17 shows a Thomson scatter frequency spectrum obtained under single-frequency pumping which illustrates this point. It is important to distinguish between beat wave mode coupling and the mode coupling of SRS-produced electron plasma waves illustrated in Fig. 17. Since the spectrum in Fig. 17 was obtained for single-frequency pumping, the primary wave is an SRS-driven plasma wave (the spectral peak which is blue shifted by $\left.\omega_{p}\right)$. The red-shifted peak arises when the $k=k_{i}$ primary wave undergoes an $m=-2$ coupling to the $k=-k_{i}$ mode.

Due to the complexity and interdependencies of the many modes and excitation mechanisms it is not always possible to decisively attribute a given experimentally observed mode to one particular mechanism. To further our understanding of the excitation of the beat wave and coupled modes we have employed computer simulations. The results of these simulations are given in Section $\mathrm{V}$.

\section{Computer Simulations of Beat Wave Mode COUPLING}

Use has been made of computer simulations to further corroborate the theoretical predictions and experimental observations of the mode coupling process and the coupled mode spectrum. Particular attention has been directed toward the study of the electrostatic $k$ spectrum and the observation of thermal effects.

These simulations were conducted using the particle code WAVE [28]. WAVE is a fully relativistic electromagnetic PIC code. The simulations were carried out in essentially one spatial dimension on a $2 \times 1500$ grid of normalized dimensions $1 \times 100$ (normalized to $c / \omega_{p 0}$ ). The code calculates and increments the electrons' positions and velocities for each time step. The positions and 
velocities are then used to calculate the new charge and current densities. The resulting fields and forces on the particles (electrons) are then calculated and the process is continued for the allotted number of time steps for which the simulation is to be run. In all simulations reported on here a total of 32000 electrons were used. To model the density ripple the electrons and a fixed ion background were initialized at time $t=0$ with a sinusoidal ripple.

Before presenting the results of the simulations it is useful to establish a workable notation and describe the normalization. In the simulations, the laser pump is incident from the left along the $x$ axis and is polarized along $\hat{y}$. Distances are normalized to the collisionless skin depth $c / \omega_{p 0}$ ( $c$ is the velocity of light in vacuum) frequencies are normalized to the background (excluding the ripple) plasma frequency $\omega_{p 0}$, and velocities are normalized to $c$. The $k$ 's are normalized to $\omega_{p 0} / c$ and are denoted by " $k x$ " , in the figures (longitudinal wavenumber). For display purposes the wavenumbers are given in units of "mode number," the number of wavelengths of a given sinusoidal mode that will fit within the simulation box: $k x$ (m.n.) $=k L / 2 \pi$ where $k$ and $L$ are given in units of $\omega_{p 0} / c$ and $c / \omega_{p 0}$, respectively. Because $L$ was chosen to be $100 c / \omega_{p 0}$ for the simulations reported on here, $k($ m.n. $)=15.9 k\left(\omega_{p 0} / c\right)$.

To model beat excitation one wants $\Delta \omega_{\text {lasers }}=\omega_{p 0}$. In our simulations, the laser frequencies were chosen to be $\omega_{1}=5, \omega_{2}=4$. In simulation units, the dispersion relation for light waves in a plasma is

$$
\omega_{1,2}^{2}=1+k_{1,2}^{2} \text {. }
$$

The chosen laser frequencies give a plasma wavenumber of $k_{p}=(24)^{1 / 2}-(15)^{1 / 2}=1.03$. We were interested in a ripple of wavenumber $k_{i}>k_{p}$. For the simulations presented, the ripple wavenumber $k_{i}$ was chosen to be either $2(24)^{1 / 2}$ or $1.2(24)^{1 / 2}$.

\section{A. Observations of Mode Coupling}

Fig. 18 shows the results of one simulation with $\epsilon=$ $0.25, p=0.508, \alpha_{1}=\alpha_{2}=0.05$, and $k_{i}=2(24)^{1 / 2}$. The simulation was set up to record plasma parameters every $\Delta\left(\omega_{p 0} t\right)=30$ (every 500 time steps). At $\omega_{p 0} t=0$ the system is initialized with the above parameters. At $\omega_{p 0} t=60$ the beat wave $\left(\Psi_{0}\right)$ and first $\left(\Psi_{ \pm 1}\right)$ coupled modes are seen to be well established above the noise. The modes are discrete in $k$ as expected from theory. At $\omega_{p 0} t=90$ the coupled modes and beat wave have continued to grow and the electron distribution function begins to show evidence of electron heating in the longitudinal direction as the waves begin to trap background electrons. The heating is anisotropic in $v_{x}$ because the right-propagating coupled mode $\left(\Psi_{+1}\right)$ has a lower phase velocity and can more efficiently trap background electrons than the somewhat faster $\left(\Psi_{-1}\right)$ mode which travels to the left. Note that "heating" also arises due to the quiver motion of the electrons in the beat wave electric field even though no appreciable fraction of the electrons can be trapped by a beat wave traveling with $v_{\phi} \cong c$. At $\omega_{p 0} t=120$ the second coupled modes are observed to emerge from the noise at precisely the predicted discrete wavenumbers. As time progresses the plasma continues to heat due to waveparticle interactions between the first and second coupled modes and the plasma electrons. Eventually ( $\omega_{p 0} t=210$, 240 ) the beat wave saturates and the coupled modes begin to decrease in amplitude due to the increase in Landau damping and the decrease in the coupling efficiency between the fixed frequency driver and the now frequencyshifted Bohm-Gross $\Psi_{+1}$ and $\Psi_{+2}$ modes. The suppression by damping of the higher $k$ (short wavelength) components is evident in both the electric field waveforms and the $k$ spectra where the amplitudes of the $\Psi_{+1}$ and $\Psi_{+2}$ modes have fallen below the noise level.

\section{B. Self-Stabilization of Saturation by Mode Coupling}

In Section II it was postulated that saturation of the beat wave could be avoided by thermally quenching the coupling process. If the plasma is heated to a sufficient temperature the Bohm-Gross frequency shift of the $\Psi_{ \pm 1}$ modes is so large that the frequency difference between the modes and the driver cannot be accommodated by the variation in $\omega_{p}(x)$ in the density ripple [7], [8]. In this case the coupled modes cease to be excited and no longer provide a sink for beat wave energy. In the absense of those losses, the mode coupling saturation mechanism is defeated and the beat wave amplitude is limited only by relativistic detuning.

Fig. 19 shows the results of a simulation with $\epsilon=0.1$, $\alpha_{1}=\alpha_{2}=0.05$, and $k_{i}=1.2(24)^{1 / 2}$. In addition, the electrons were initialized at $\omega_{p 0} t=0$ with zero thermal motion to effect a "cold start." At $\omega_{p 0} t=60$ both the electric field waveform and the $k$ spectrum show strong mode coupling while the electron distribution function indicates that the plasma is still quite cold $\left(k_{i} \lambda_{d} \leq 0.1\right)$. At $\omega_{p 0} t=120$ the electron distribution shows that significant plasma heating has occurred and the electric field $k$ spectrum shows appreciable suppression of the coupled modes while the beat wave has continued to grow. Spatially, the electric field waveform shows that at the back of the laser pulse, where the plasma has had longer time to heat, the waveform is devoid of high- $k$ components. This is because the high- $k$ components have Landau damped away and are no longer driven by the mode coupling process due to the thermally enhanced Bohm-Gross frequency shift of the heated plasma. At $\omega_{p 0} t=180$ the plasma temperature has stabilized and virtually all traces of the coupled mode components of the wavenumber spectrum have vanished. At this time the beat wave has resumed its growth unabated by energy channeling to the coupled modes.

\section{Temporal Evolution of Beat Wave and Coupled Modes}

Thus far the simulations have been in good agreement with the theory. In particular, discreteness in wavenumber of the coupled mode spectrum and stabilization of the beat wave mode coupling saturation mechanism by ther- 


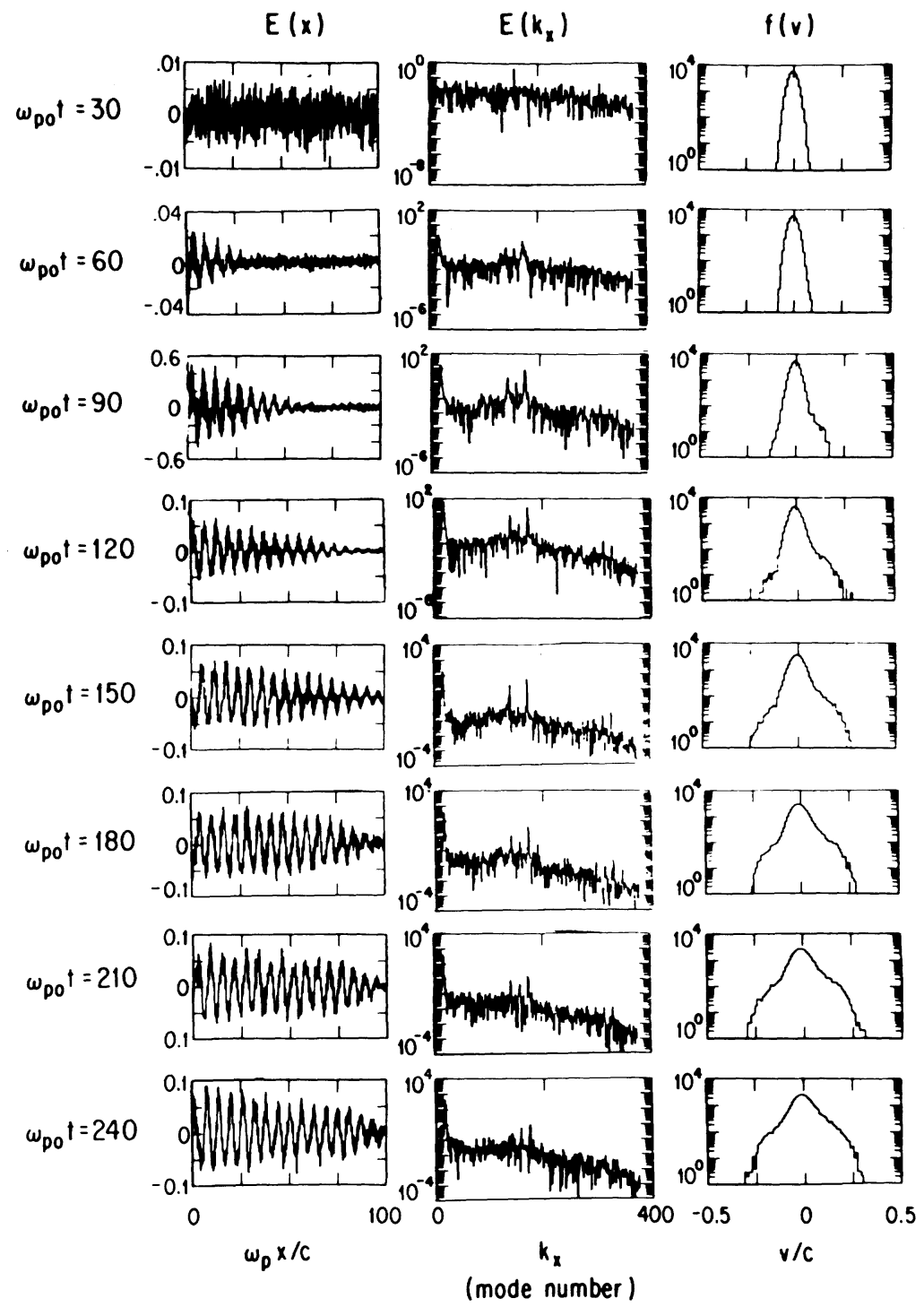

Fig. 18. Plots of $E_{\text {long. }}(x), E_{\text {long. }}\left(k_{x}\right)$, and the electron distribution function $F(v)$ obtained from simulation $\left(\epsilon=0.25, \alpha_{1}=\alpha_{2}=0.05, k_{i}=\right.$ $2(24)^{1 / 2}$, and $\left.p=0.508\right)$. $E_{\text {long. }}(x)$ is normalized to $m c \omega_{p 0} / e$. Refer to the text for a description of the normalizations used for the $x$ and $k x$ axes.

mal quenching have been demonstrated. To further explore the applicability of the warm plasma mode coupling theory it is of interest to test the theoretical predictions of the saturation amplitudes and saturation times of the beat wave and coupled modes using the results of computer simulations. However, before doing so it is worthwhile to note an inherent limitation in the above simulation results.

When attempting to follow the growth in time of the beat wave and coupled modes one is naturally tempted to use the peak heights of the various modes obtained from the electrostatic $k$ spectra (e.g., Fig. 18). The difficulty lies in the fact that the $k$ spectrum represents an integration over the length of the simulation box. At later times, after the wave has progressed further across the box, the $k$ spectra naturally reflect the fact that the box contains more of a given mode. In an absolute sense, the wave does not really contain more of that mode. Thus, as in the $\omega_{p 0} t=120$ case of Fig. 20, the amplitudes of the beat wave and coupled mode spectral peaks cannot be straightforwardly interpreted and one cannot expect to unambiguously determine a growth curve for the beat wave or coupled modes.

To overcome this difficulty we performed simulations which sampled the electric field only in the left quarter of the simulation box (the first 375 grid points, $x=0-25$ ). Thus, for $\omega_{p 0} t \geqslant 25$, the field sampling region is completely filled with the beat wave field. There is still some inherent temporal averaging within the field sampling region because the leading edge of the beat wave has had less time to grow than the trailing edge.

In Fig. 20 we make a direct comparison of the temporal evolution of the beat wave $\left(\Psi_{0}\right)$ and first and second $\left(\Psi_{ \pm 1}, \Psi_{ \pm 2}\right.$ ) coupled modes obtained from simulation and the warm plasma mode coupling theory. Fig. 20(a) shows 


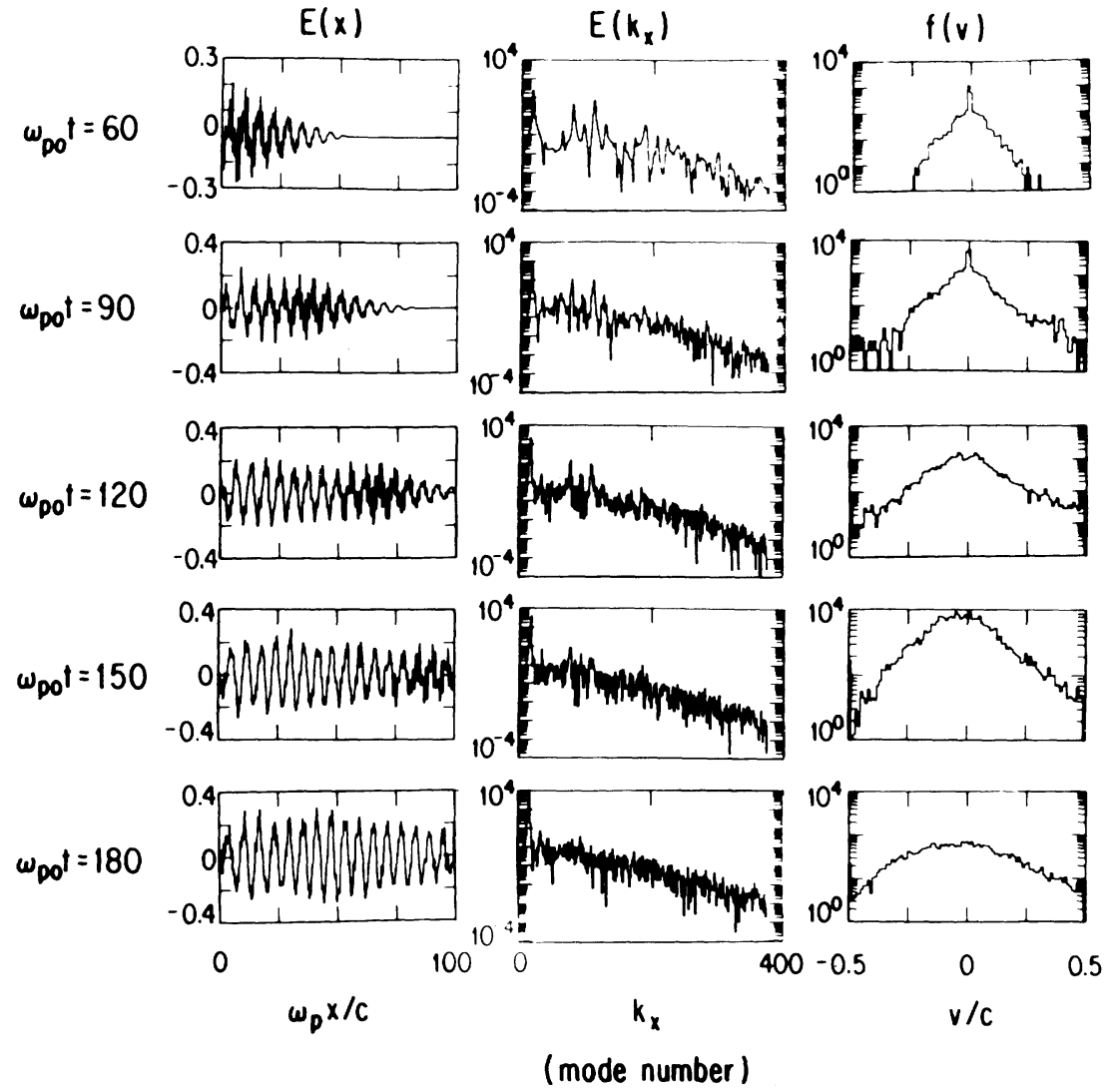

Fig. 19. Plots of $E_{\text {long. }}(x), E_{\text {long. }}(k x)$, and the electron distribution function $F(v)$ obtained from simulation $\left(\epsilon=0.1, \alpha_{1}=\alpha_{2}=0.05, k_{i}=\right.$ $1.2(24)^{1 / 2}$, and $\left.p(t=0)=0\right)$. The electrons were initialized with zero thermal motion. $E_{\text {long. }}(x)$ is normalized to $m c \omega_{p 0} / e$. Refer to the text for a description of the normalizations used for the $x$ and $k x$ axes.
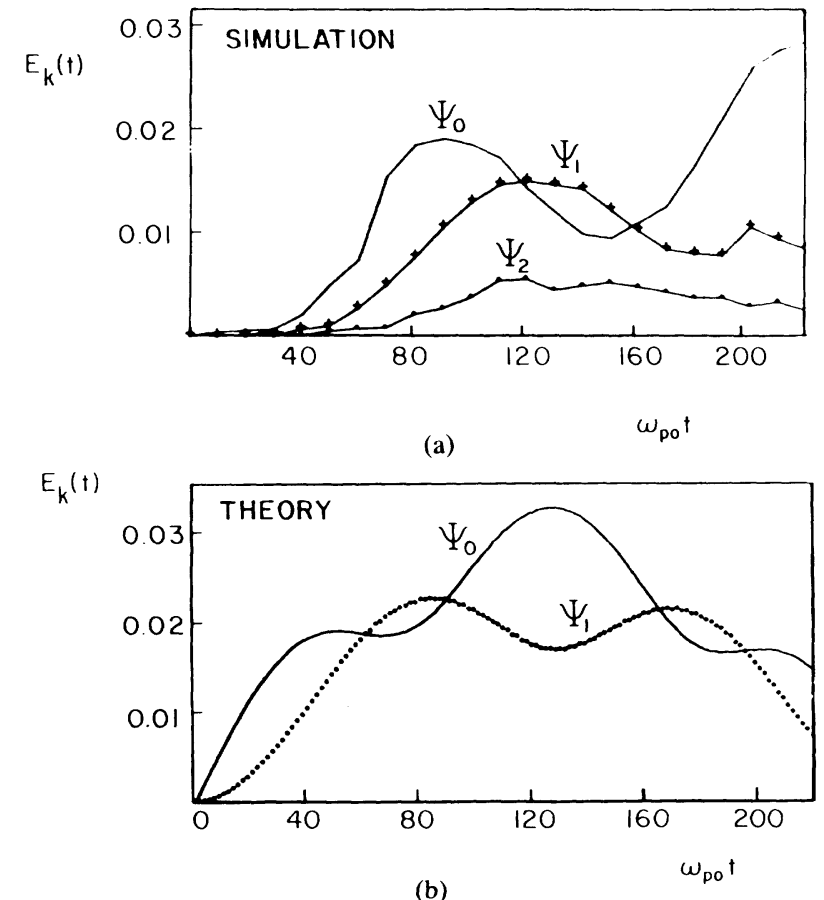

Fig. 20. Comparison of the beat wave ( $\left.\Psi_{0}\right)$ and first $\left(\Psi_{ \pm 1}\right)$ coupled mode growth curves obtained from (a) simulation, and (b) theory. In both cases $E_{k}$ is normalized to $m c \omega_{p 0} / e$. Shown also for the simulation is the growth curve for the $\Psi_{ \pm 2}$ mode, not included in the warm-plasma theory. the growth curve obtained from the simulation for $\epsilon=$ $0.1, p=0.466$, and $\alpha_{1}=\alpha_{2}=0.05$, and $k_{i}=$ $1.2(24)^{1 / 2}$. The electrostatic field at the left-hand side of the simulation box was sampled every $\Delta\left(\omega_{p 0} t\right)=10$ (every 167 time steps) and the peak amplitude of the beat wave $\left(\Psi_{0}\right)$ and first $\left(\Psi_{ \pm 1}, \Psi_{ \pm 2}\right)$ two coupled modes were recorded. The peak amplitudes were then plotted versus $\omega_{p 0} t$ as shown (the points plotted for the $\Psi_{ \pm 1}$ and $\Psi_{ \pm 2}$ modes represent the averages $\frac{1}{2}\left[E\left(\Psi_{1}\right)+E\left(\Psi_{-1}\right)\right]$ and $\frac{1}{2}\left[E\left(\Psi_{2}\right)+E\left(\Psi_{-2}\right)\right]$, respectively). Similarly, the theoretical curves in Fig. 20(b) were generated by sampling the spectra of (23a) and (23b) at regular intervals and plotting the mode amplitudes versus $\omega_{p 0} t$. It is immediately evident that early in time the warm-plasma mode coupling theory predicts the qualitative behavior of the simulation fields with remarkable accuracy. The beat wave is seen to grow first in time. A short time later the first coupled mode begins to grow. After growth of the first coupled mode has stabilized, the beat wave growth is curtailed and a temporally local beat wave maximum is reached as energy is coupled out of the beat wave and into the first coupled mode. This in turn leads to diminished growth of the first coupled mode.

Several features of the temporal evolution curves are worthy of comment early in this discussion. It is apparent 


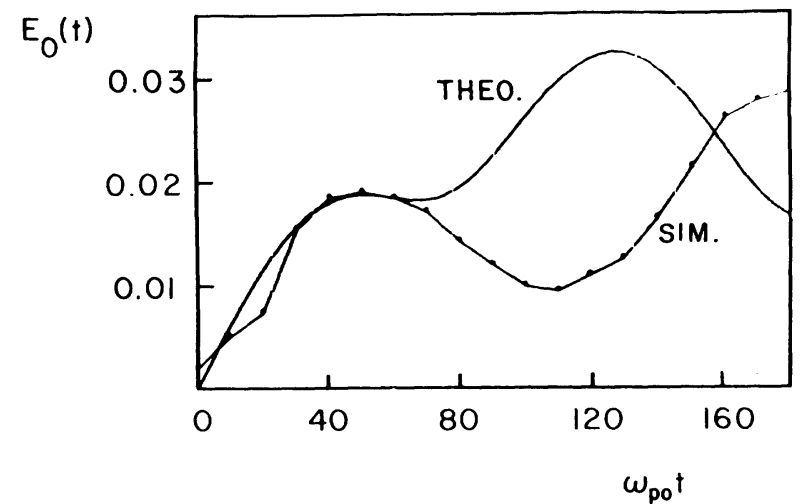

(a)

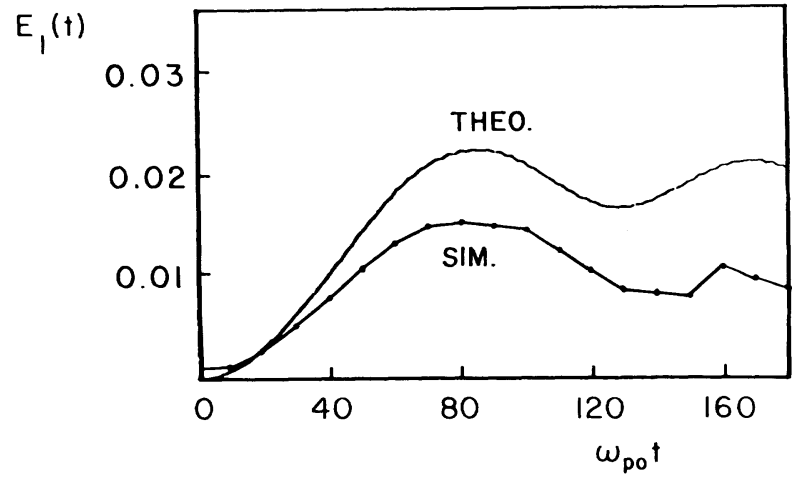

(b)

Fig. 21. Direct comparison of the theory and simulation growth curves for (a) the beat wave ( $\Psi_{0}$ mode), and (b) the first $\left(\Psi_{ \pm 1}\right)$ coupled mode. The simulation growth curves have been offset to the left by $40 \omega_{p 0} t$ to compensate for the laser rise time and the "fill time" of the simulation field diagnostic region. The fields are normalized to $m c \omega_{p 0} / e$.

that the growth of the modes in the simulation curve lags that of the theory curves by about $30-50 \omega_{p 0} t$. This behavior is expected in light of two considerations. First, the beat wave required about $25 \omega_{p 0}^{-1}$ to fill the region sampled by the field diagnostic in the simulation. Secondly, the laser rise time $\left(\omega_{p 0} t_{\text {rise }}=50\right)$ adds to the delay since the growth rates scale as $\alpha_{1} \alpha_{2}$ for early times (although rigorously shown only for the cold-plasma case it is a reasonable approximation for early times in the warm-plasma case). Although, with the theory in its present form, one cannot hope to assess completely the effects of pump rise time, it is fruitful to take these time delays into consideration when comparing the theory and simulation growth curves. In Fig. 21 we have offset the simulation curves by $\omega_{p 0} t=40$ and separately superimposed the $\Psi_{0}$ and $\Psi_{ \pm 1}$ theory curves for comparison. The qualitative agreement is of course preserved but one now can see good agreement between theory and simulation regarding saturation times of the $\Psi_{0}$ and $\Psi_{ \pm 1}$ modes. Referring to Fig. 21 one can see that, in some sense, the term "saturation time" is not well defined. For example in the simulation curve of Fig. 21(a), the beat wave first "saturates" at $\omega_{p 0} t=50$ and then again at $\omega_{p 0} t=180$. This is of course not true saturation behavior if the beat wave continues to "step up" every $\omega_{p 0} t \cong 140$ for $\omega_{p 0} t \geqslant 180$. However, based on the theoretical predictions for $\omega_{p 0} t \geqslant 180$, the two local maxima do represent global maxima because the

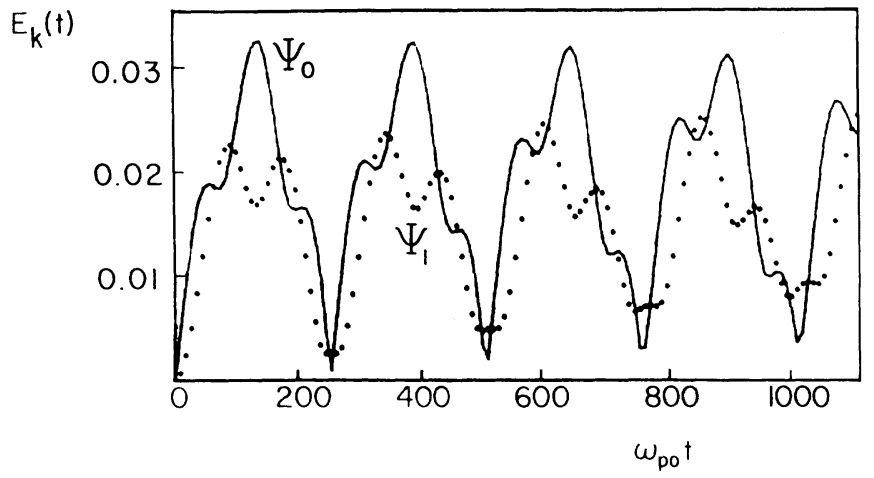

Fig. 22. Long-time behavior of the warm-plasma theory solution for the beat wave $\left(\Psi_{0}\right)$ and first $\left(\Psi_{ \pm 1}\right)$ coupled mode. The fields are normalized to $m c \omega_{p 0} / e$. The $\Psi_{0}$ and $\Psi_{ \pm 1}$ growth curves are periodic with periodicity $\cong 250 \omega_{p 0}^{-1}$. The periodic behavior illustrates the fact that the local maxima are indicative of true saturation behavior.

growth curves are periodic with periodicity $\omega_{p 0} t \cong 250$. This point is illustrated in Fig. 22 where we have plotted the warm-plasma mode amplitudes ((23a) and (23b)) out to $\omega_{p 0} t=1100$. The long-time behavior of the growth curves resembles a bumpy sine wave as one might expect upon inspection of (23a) and (23b).

It should be kept in mind that for the parameters used in the simulation and theory results presented in Figs. 2022 , the relativistic detuning saturation mechanism is not important since the condition $\alpha_{1} \alpha_{2},<(1.6 \epsilon / f(p))$ (cf. Section III) is clearly satisfied. In'addition, the time to saturation by relativistic detuning is about $500 \omega_{p 0}^{-1}$, well beyond the $\omega_{p 0} t_{\text {sat }}$ of 150 relevant to the results under study here.

The theory and simulation growth curves are in excellent agreement for both the $\Psi_{0}$ and $\Psi_{ \pm 1}$ modes (cf. Fig. 21 (a) and (b)). In particular both the theory and simulation curves exhibit the local field maximum at $\omega_{p 0} t \cong 50$ for the $\Psi_{0}$ mode (beat wave). However, in the simulation curves both the beat wave and first coupled mode are observed to diminish in amplitude significantly while the theory curves show little decay preceding the second maximum. In addition, the $\Psi_{0}$ mode is larger and the $\Psi_{ \pm 1}$ mode is smaller than for the theory curve at these later times. There are again at least two contributing factors. First, at $\omega_{p 0} t \cong 100$ weak plasma heating has occurred and to some extent enhances Landau damping of the first and second coupled modes (and higher order modes).

A second and probably more significant effect not accounted for by the theory is the excitation of the second coupled mode itself. In Fig. 23 we have reproduced the $\Psi_{0}$ and $\Psi_{ \pm 1}$ growth curves on the offset time scale (offset by $\left.40 \omega_{p 0} t\right)$. One can see that the $\Psi_{0}$ mode behavior departs most severely from the theoretical prediction at exactly the time when the $\Psi_{ \pm 2}$ mode has reached its largest amplitude. Because the warm-plasma theory of Section II does not take the higher order modes into consideration, this behavior is not unexpected. The $\Psi_{ \pm 2}$ mode and higher order modes all act as a sink of beat wave energy and further reduce its amplitude. The warm-plasma theory of Section II does not take the higher order modes into con- 


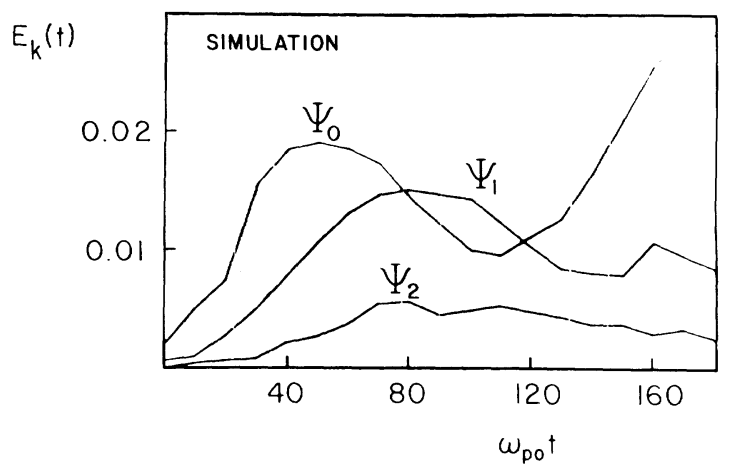

Fig. 23. Growth curves for the $\Psi_{0}, \Psi_{ \pm 1}$, and $\Psi_{ \pm 2}$ modes obtained from simulation. These curves show the exchange of energy between the various modes excited. The time scale has been offset to the left by $40 \omega_{p 0} t$ and the fields are normalized to $m c \omega_{p 0} / e$.

sideration. In their absence, it is not unreasonable to expect that the beat wave and first coupled mode sustain a higher amplitude since no higher order modes are present to absorb energy.

\section{Estimation of Beat Wave Saturation Time}

A simple law for estimating the beat wave saturation time was empirically derived by determining the beat wave saturation time from the theoretical growth curves as a function of the thermal parameter $p$ and the ripple depth $\epsilon$. The results of these measurements are shown in Fig. 24. It was found that $\omega_{p 0} t_{\text {sat }} \cong 17.5 p / \epsilon$. This law provides a reasonable estimate of $\omega_{p 0} t_{\text {sat }}$ for $p \geqslant 0.2$ since, for sufficiently hot plasmas, the ambiguities in assigning a saturation time discussed above are not encountered (cf. Fig. 3). The $\omega_{p 0} t_{\text {sat }}$ given by the law for the parameters of the simulation in Figs. 20 and 21 is in fair agreement with the results of that cool-plasma simulation $\left(\omega_{p 0} t_{\text {sat }} \cong\right.$ 100 ). A simple check of this scaling law's independence of pump amplitude was made. A simulation with all parameters identical to those used in the simulation presented in Figs. 20 and 21 was performed with a weaker laser pump ( $\alpha=0.02$ compared to $\alpha=0.05)$. The saturation time for the beat wave was found to be about the same as in the strong pump case and, if at all, is only very weakly dependent on the laser pump amplitude.

\section{E. Saturation Amplitudes}

The theoretically predicted beat wave $\left(\Psi_{0}\right)$ and first $\left(\Psi_{+1}\right)$ coupled mode saturation amplitudes are given by (25a) and (25c). For the parameters used in the simulation of Fig. $21\left(\epsilon=0.1, p=0.466, \alpha_{1}=\alpha_{2}=0.05\right.$, and $k_{i}$ $\left.=1.2(24)^{1 / 2}\right)$ the expected saturation amplitude for the $\Psi_{0} \Psi_{ \pm 1}$ modes are 0.032 and 0.025 , respectively. Referring to Fig. 23, the $\Psi_{0}$ and $\Psi_{ \pm 1}$ saturation amplitudes observed in the simulations were 0.028 and 0.015 , respectively. While the saturation amplitude of the $\Psi_{0}$ mode is in excellent agreement with the theory, the saturation amplitude of the $\Psi_{ \pm 1}$ mode observed in the simulation is only about 60 percent of its predicted value. This is possibly due to the fact that, at the time of saturation of the $\Psi_{ \pm 1}$ mode (cf. Fig. 23), the $\Psi_{ \pm 2}$ mode amplitude is at its max-

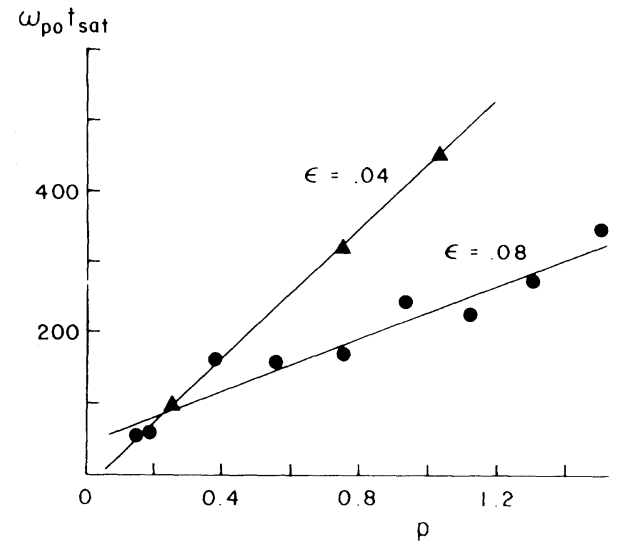

Fig. 24. Plots of $\omega_{p 0} t_{\text {sat }}$ (beat wave) versus $p$ for two different ripple depths $\epsilon$. These results were obtained from the warm-plasma mode coupling solutions (23) and (24). For $p \geqslant 0.2$ the empirically derived law, $\omega_{p 0} t_{\text {sat }}$ $\cong 17.5 \mathrm{p} / \epsilon$, provides a reasonable estimate of the beat wave saturation time.

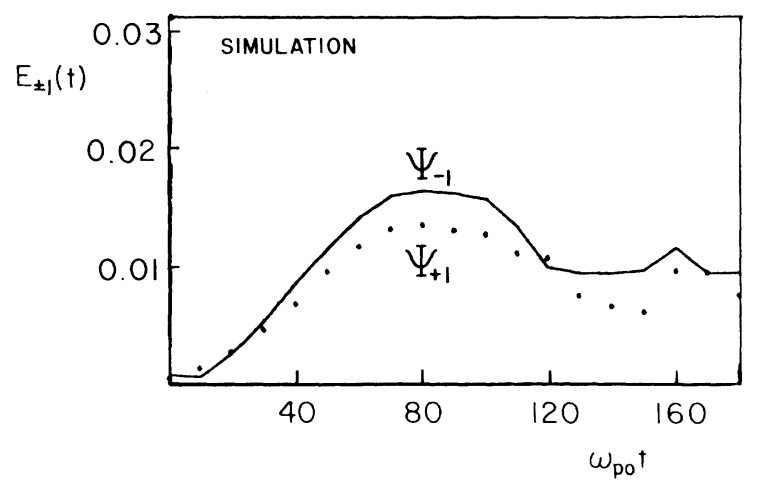

Fig. 25. Growth curves for the $\Psi_{+1}$ and $\Psi_{-1}$ modes obtained from simulation. The higher phase velocity $\Psi_{-}$, mode maintains a slightly higher amplitude than the slower $\Psi_{+1}$ mode.

imum and presents the maximum possible energy sink to both the $\Psi_{0}$ and $\Psi_{ \pm 1}$ modes. In addition, the higher order modes (not shown) further act to reduce the amplitude of the $\Psi_{0}$ and $\Psi_{ \pm 1}$ modes in the simulation.

The excitation to finite amplitudes of the higher order modes is an obvious point of departure of the experiment from the idealized theoretical model. Interestingly, the experimentally measured amplitudes of the $\Psi_{0}$ and $\Psi_{ \pm 1}$ modes (cf. Fig. 14) also fall below their theoretically predicted values. In addition, the Thomson scatter spectra of Section III consistently showed that the $\Psi_{+n}$ modes had higher amplitudes than the $\Psi_{-n}$ modes $(n>0)$. In Fig. 25 we have plotted the $\Psi_{+1}$ and $\Psi_{-1}$ growth curves obtained from simulation (the same curves which, when averaged, were presented in Figs. 20, 21, and 23). The growth curves exhibit the opposite behavior as expected from the experimental measurements. One can imagine in the simulations, because the $\Psi_{+1}$ mode is significantly slower ( $\cong 30$ percent) than the smaller- $k \Psi_{-1}$ mode, that Landau damping has a stronger impact on the $\Psi_{+1}$ mode than on the $\Psi_{-1}$ mode and yields a smaller $\Psi_{+1}$ mode amplitude. By contrast in the experiment, the $k$ 's of the $\Psi_{+1}$ and $\Psi_{-1}$ modes are very nearly equal and differential Landau damping could not explain the difference in am- 
plitudes even if the $\Psi_{+1}$ mode had been observed to be smaller (which it was not). It is more likely in the experiment that CPOM is a more important consideration in resolving the question of the relative amplitudes of the $\Psi_{ \pm 1}$ and $\Psi_{-1}$ coupled modes.

\section{SUMmary}

A simple theoretical model for beat excitation of electron plasma waves in a rippled density plasma has been presented. The model predicts a new beat wave saturation mechanism. In cold plasmas the beat wave saturates because an infinite number of longer- $k$ secondary electrostatic modes are excited. These modes have frequencies equal to $\omega_{p 0}$ (the background plasma frequency) but their wavenumbers are equal to the beat wave wavenumber $k_{p}$ plus multiples of the ripple wavenumber $k_{i}$. The ripple is commonly encountered in experiments in the form of an ion-acoustic wave driven by the stimulated Brillouin instability. These coupled modes divert laser pump energy from the beat wave. When the rate at which pump energy is supplied to the beat wave equals the rate at which energy is deposited in the coupled modes, the beat wave saturates. The saturation amplitude (normalized to the cold plasma wave breaking field $m c \omega_{p 0} / e$ ) in cold plasmas is given by $E_{\text {sat }}$ (cold) $=\alpha_{1} \alpha_{2} / \epsilon$ where $\alpha_{j}=$ $e E_{j} / m \omega_{j} c$ parameterizes the laser intensity and $\epsilon$ is a measure of the ripple depth.

In warm plasmas the electrostatic coupled mode spectrum is limited in the number of excited modes because only those modes whose Bohm-Gross frequency shifts do not exceed the variation of $\omega_{p 0}(x)$ in the density ripple are excited. Because the number of coupled modes is limited, the beat wave saturates at a higher amplitude. In warm plasmas, the saturation amplitude is given by $E_{\text {sat }}($ warm $)=\alpha_{1} \alpha_{2} f(p) / \epsilon$ where $f(p)=p+\left(1+p^{2}\right)$ $(2+p)^{-1 / 2}$ is a thermal enhancement factor and $p$ is a thermal parameter defined by $p=3\left(k_{i} \lambda_{d}\right)^{2} / \epsilon$.

The relevance of the mode coupling saturation mechanism can be assessed by comparing the mode coupling beat wave saturation amplitude with that expected for relativistic detuning. One finds that saturation by mode coupling dominates over that due to relativistic detuning whenever $\alpha_{1} \alpha_{2}<(1.6 \epsilon / f(p))^{3 / 2}$. For example in the case of a modest ripple size of $\epsilon=0.04$ and $\alpha_{1}=\alpha_{2}$, saturation by mode coupling dominates unless $\alpha_{j}>0.04$ or, in the case of a $\mathrm{CO}_{2}$ laser, unless $I\left(\mathrm{CO}_{2}\right)>4 \times 10^{13}$ $\mathrm{W} / \mathrm{cm}^{2}$. Although this requirement may be altered somewhat when laser rise times are considered, it is apparent that the mode coupling saturation mechanism can be quite important in moderate-intensity or shorter-wavelength experiments.

The coupled mode spectrum itself is of interest from a basic plasma physics standpoint, irrespective of the beat wave. Experiments which were designed to study and characterize the $\omega$ and $k$ spectrum of the coupled modes as well as that of the beat wave were performed. These experiments were performed in $10^{17}-\mathrm{cm}^{-3}$ density plasma using the 10.6- and 9.6- $\mu \mathrm{m}$ lines of 2-ns pulsewidth $\mathrm{CO}_{2}$ laser capable of delivering about $10^{13} \mathrm{~W} / \mathrm{cm}^{2}$ at its focus. Detailed measurements employing ruby laser Thomson scattering were made. The measured electrostatic $\omega$ and $k$ spectrum agreed well with the theory. In addition, the measured coupled mode amplitudes were consistent with the mode coupling saturation model. Although the coupled modes were conclusively observed, the experimental parameters were such that the actual beat wave saturation amplitude measured was consistent with both the mode coupling and relativistic detuning saturation models.

To further corroborate the theoretical predictions and experimental measurements, particle simulations were performed using the PIC code WAVE. Particular attention was directed toward the observation of the beat wave mode coupling saturation mechanism and the theoretically predicted properties of the coupled mode $k$-spectrum.

Early simulations verified the excitation of the beat wave and coupled modes at precisely the theoretically predicted wavenumbers. In addition, by following and recording the temporal development of the beat wave and coupled modes we were able to compare theory and simulation directly for the same parameters. The growth curves for the beat wave and coupled modes are in excellent agreement at early times with regard to saturation times and saturation amplitudes. At later times the deviation in behavior of the theory and simulation growth curves becomes evident as the contributions of the higher order coupled modes, not included in the warm-plasma model, become more and more important.

In cold-plasma long-pulse experiments, the mode coupling saturation mechanism can undergo self-stabilization if the plasma is heated to the point where the Bohm-Gross frequency shift of the first coupled mode is sufficiently large to detune them from the fixed frequency driver. This self-stabilization mechanism was observed in one simulation whose electrons were initialized at $t=0$ with zero thermal motion. For early times the beat wave grew much as predicted by the cold-plasma theory and the $k$ spectrum clearly indicated the presence of the coupled modes. However, at later times we observed abrupt quenching of the coupling concomitant with the onset of particle heating observed in the electron distribution function and in electron phase space.

\section{ACKNOWLEDGMENT}

The authors thank Prof. J. M. Dawson and Prof. F. F. Chen for useful discussions.

\section{REFERENCES}

[1] T. Tajima and J. M. Dawson, Phys. Rev. Lett., vol. 43, p. 267, 1969.

[2] C. Joshi et al., Nature, vol. 311, p. 256, 1984.

[3] N. M. Kroll, A. Ron, and N. Rostoker, Phys. Rev. Lett., vol. 13, p. 83, 1964.

[4] D. Montgomery, Physica (Utrecht), vol. 31, p. 693, 1965.

[5] R. A. Stern and N. Tzoar, Phys. Rev. Lett., vol. 16, p. 785, 1966.

[6] M. N. Rosenbluth and C. S. Liu, Phys. Rev. Lett., vol. 29, p. 701, 1972.

[7] W. L. Kruer, Phys. Fluids, vol. 15, p. 2423, 1972.

[8] P. K. Kaw, A. T. Lin, and J. M. Dawson, Phys. Fluids, vol. 16, p. 1967, 1973. 
[9] C. M. Tang and P. Sprangle, Appl. Phys. Lett., vol. 45, p. 375, 1984. [10] J. M. Dawson, Phys. Rev., vol. 113, p. 383, 1959.

[11] B. Amini and F. F. Chen, Phys. Rev. Lett., vol. 53, p. 1441, 1984.

[12] C. Aldrich et al., Plasma Phys. Controlled Fusion, vol. 10, p. 1, 1986.

[13] C. Clayton, C. Joshi, and F. F. Chen, Laser Interactions and Related Plasma Phenomena, vol. 6. New York: Plenum.

[14] C. J. Walsh, D. M. Villeneuve, and H. A. Baldis, Phys. Rev. Lett., vol. 53, p. $1445,1984$.

[15] D. M. Villeneuve, C. J. Walsh, and H. A. Baldis, Phys. Rev. Lett., vol. 28, p. 1591, 1985.

[16] S. J. Kartunnen, Plasma Phys., vol. 22, p. 251, 1980.

[17] D. E. Evans and J. Katzenstein, Rep. Prog. Phys., vol. 32, p. 207, 1969.

[18] C. E. Clayton, C. Darrow, and C. Joshi, Appl. Optics, vol. 24, p. 2823,1985 .
[19] C. Clayton, C. Joshi, C. Darrow, and D. Umstadter, Phys. Rev. Lett., vol. 55, p. 1652, 1985.

[20] C. Darrow et al., Phys. Rev. Lett., vol. 56, p. 2629, 1986.

[21] D. W. Forslund, J. M. Kindel, and E. L. Lindman, Phys. Rev. Lett., vol. 30, p. $739,1973$.

[22] D. W. Forslund, J. M. Kindel, and E. L. Lindman, Phys. Fluids, vol. 18 , p. 1002, 1975.

[23] D. W. Forslund, J. M. Kindel, and E. L. Lindman, Phys. Fluids, vol. 18 , p. $1017,1975$.

[24] L. D. Landau and E. M. Lifshitz, Electrodynamics of Continuous Media. New York: Pergamon.

[25] R. E. Slusher and C. M. Surko, Phys. Fluids, vol. 23, p. 472, 1980.

[26] F. Martin and T. W. Johnston, Phys. Rev. Lett., vol. 55, p. 1651, 1985.

[27] C. Darrow, Ph.D. dissertation, Univ. Calif., Los Angeles, CA, 1986.

[28] R. L. Morse and C. W. Neilson, Phys. Fluids, vol. 14, p. 830, 1971. 